\title{
Novel Observer-Based Suboptimal Digital Tracker for a Class of Time-Delay Singular Systems
}

\author{
Nien-Tsu Hu, ${ }^{1}$ Ter-Feng $W u,{ }^{2}$ Sendren Sheng-Dun $X u,{ }^{3}$ and Hsu-Chih Huang ${ }^{2}$ \\ ${ }^{1}$ Integrated Logistical Support Center, Chung-Shan Institute of Science and Technology, Taoyuan 32599, Taiwan \\ ${ }^{2}$ Department of Electrical Engineering, National Ilan University, Ilan 26047, Taiwan \\ ${ }^{3}$ Graduate Institute of Automation and Control, National Taiwan University of Science and Technology, Taipei 10607, Taiwan
}

Correspondence should be addressed to Ter-Feng Wu; tfwu@niu.edu.tw

Received 12 July 2013; Accepted 28 September 2013

Academic Editor: Baocang Ding

Copyright (C) 2013 Nien-Tsu Hu et al. This is an open access article distributed under the Creative Commons Attribution License, which permits unrestricted use, distribution, and reproduction in any medium, provided the original work is properly cited.

\begin{abstract}
This paper presents a novel suboptimal digital tracker for a class of time-delay singular systems. First, some existing techniques are utilized to obtain an equivalent regular time-delay system, which has a direct transmission term from input to output. The equivalent regular time-delay system is important as it enables the optimal control theory to be conveniently combined with the digital redesign approach. The linear quadratic performance index, specified in the continuous-time domain, can be discretized into an equivalent decoupled discrete-time performance index using the newly developed extended delay-free model. Additionally, although the extended delay-free model is large, its advantage is the elimination of all delay terms (which included a new extended state vector), simplifying the proposed approach. As a result, the proposed approach can be applied to a class of time-delay singular systems. An illustrative example demonstrates the effectiveness of the proposed design methodology.
\end{abstract}

\section{Introduction}

The singular systems naturally arise in describing large-scale systems, and there are several examples occurring in power and interconnected systems. In general, an interconnection of state variable subsystems is conveniently described as a singular system, even though an overall state space representation may not even exist. Over the past decades, much research into singular systems has solved many complex problems concerning, for example, the stability [1-4], impulsive modes [5], controllability, observability [6], and the sufficient and necessary conditions for the impulse controllability and observability of time-varying singular systems [7-11]. However, the main purpose of such work is either to stabilize the singular system or to prove its controllability and observability. Here, the key note of this paper is about tracking the issue.

This investigation considers a time-delay system. The overwhelming majority of practical control systems are described by continuous-time settings with input, output, and state time delays. Those delays arise from inherent physical phenomena and are commonly encountered in various engineering systems. Several authors [12-15] have studied the linear quadratic optimal analog controllers for the analog system with input and state delays. Recently, robust control and filtering for both continuous-time and discretetime nominal/uncertain systems with time delays have been thoroughly studied by Mahmoud [16]. Despite much progress in both analog control theory and digital control theory over the last few decades, effective digital control of analog plants with input and state delays (input-state delayed hybrid control systems) is still being developed [17, 18].

The objective of this paper is to develop a novel observerbased suboptimal digital tracker for a class of time-delay singular systems. The developed digital tracker can make the outputs of the digitally controlled time-delay singular system track the desired reference signals. First, the timedelay singular system is converted into a regular time-delay system that contains a direct transmission term from input to output. Then, for effective utilization of the well-developed discrete-time optimal control theory for a regular time-delay system, it is converted into a new extended discrete delayfree model. The performance cost function is discretized using the extended discrete delay-free model. When the 
states of the continuous time-delay singular system are not available for measurements, a suboptimal digital observer for the original continuous time-delay singular system is constructed by using the duality of the digital redesign technique for the controller and the digital-to-analog model conversion technique [19]. As a result, the proposed novel observer-based suboptimal digital tracker is able to make the output of the digitally controlled analog time-delay system track the desired reference signals.

The rest of the paper is organized as follows. Section 2 presents the problem description and preliminary results. Section 3 presents the novel optimal tracker and a novel observer-based suboptimal tracker for the time-delay singular system and proposes a systematic design methodology for designing a set of high-performance trackers for a class of time-delay systems. Finally, an illustrative example is given to demonstrate the effectiveness of the proposed approach.

\section{Problem Description and Preliminaries}

2.1. Problem Description. Consider the following continuous time-delay singular system:

$$
\begin{gathered}
E \dot{x}_{c}(t)=A x_{c}(t)+\sum_{i=1}^{N_{1}} \widehat{A}_{i} x_{c}\left(t-\tau_{s, i}\right)+\sum_{j=1}^{N_{2}} B_{j} u_{c}\left(t-\tau_{i, j}\right), \\
y_{c}(t)=C x_{c}\left(t-\tau_{o}\right),
\end{gathered}
$$

where $x_{c}(t) \in \Re^{n}$ is the state vector, $u_{c}(t) \in \mathfrak{R}^{m}$ is the control input vector, and $y_{c}(t) \in \mathfrak{R}^{p}$ is the output vector. $E, A, \widehat{A}_{i}$, $B_{j}$, and $C$ are known constant system matrices of appropriate dimensions and $E$ is a singular matrix. The corresponding state time delay $\tau_{s, i}, i=1,2, \ldots, N_{1}$, input time delay $\tau_{i, j}$, $j=1,2, \ldots, N_{2}$, and output time delay $\tau_{o}$ are assumed to be known.

The continuous time-delay singular system (la) and (1b) may be in impulsive modes. Directly designing the controller or observer for (1a) and (1b) is very difficult because impulsive modes are uncontrollable. To solve this problem, the regular pencil, the standard pencil, and the preliminary feedback control methods are used to eliminate impulsive modes and then obtain an equivalent regular time-delay system that can be applied to the original continuous time-delay singular system (1a) and (1b). The following section systematically develops the design of the novel controller and observer using the equivalent regular time-delay system.

2.2. Preliminaries. The regular pencil and standard pencil are defined below.

Definition 1 (regular pencil [20]). Let $E$ and $A$ be two square constant matrices. If $\operatorname{det}(s E-A) \neq 0$, for all $s$, then $(s E-A)$ is called a regular pencil.

Definition 2 (standard pencil [21]). Let $\left(s E_{n}-A_{n}\right)$ be a regular pencil. If there exists scalars $\alpha$ and $\beta$ such that $\alpha E_{n}+\beta A_{n}=I_{n}$, then $\left(s E_{n}-A_{n}\right)$ is called a standard pencil.

Notably, for any regular pencil, $(s E-A)$ can be easily transformed into a standard pencil by multiplying $(\alpha E+\beta A)^{-1}$ to $E$ and $A$, respectively, where $\alpha$ and $\beta$ are scalars such that $\operatorname{det}(\alpha E+\beta A) \neq 0$. Therefore, the matrix coefficients of a standard pencil $\left(s E_{n}-A_{n}\right)$ become

$$
\begin{aligned}
& E_{n}=\left(\alpha E_{r}+\beta A\right)^{-1} E, \\
& A_{n}=(\alpha E+\beta A)^{-1} A .
\end{aligned}
$$

The modified system retains its state vector $x_{c}(t)$ and the matrices $E_{n}$ and $A_{n}$ have the following nice properties.

Lemma 3 (see [22]). Consider

(a) $E_{n} A_{n}=A_{n} E_{n}$, meaning that $E_{n}$ and $A_{n}$ commute each other;

(b) $E_{n}$ and $A_{n}$ have the same eigenspaces.

The above properties enable a singular system to be decomposed into a reduced-order regular subsystem and a nondynamic subsystem.

\section{Main Results}

3.1. Decomposition of Time-Delay Singular System. By (2a) and (2b) the regular pencil $(s E-A)$ can be transformed into a standard pencil $\left(s E_{n}-A_{n}\right)$. Notably since $E_{n}$ is a singular matrix, which has at least one zero eigenvalue, $\beta$ cannot be equal to zero. Hence, multiplying (1a) by $(\alpha E+\beta A)^{-1}$ can yield the following equation:

$$
E_{n} \dot{x}_{c}(t)=A_{n} x_{c}(t)+\sum_{i=1}^{N_{1}} \widehat{A}_{n, i} x_{c}\left(t-\tau_{s, i}\right)+\sum_{j=1}^{N_{2}} B_{n, j} u_{c}\left(t-\tau_{i, j}\right),
$$

where

$$
\begin{aligned}
E_{n} & =(\alpha E+\beta A)^{-1} E, \\
\widehat{A}_{n, i} & =(\alpha E+\beta A)^{-1} \widehat{A}_{i}, \\
B_{n, j} & =(\alpha E+\beta A)^{-1} B_{j} .
\end{aligned}
$$

Since $\alpha E_{n}+\beta A_{n}=I_{n}$, the pencil $\left(s E_{n}-A_{n}\right)$ is a standard one, and has the properties that are mentioned in Lemma 3. To decompose system (3), the state $x_{c}(t)$ is converted into $\bar{x}_{c}(t)$ by

$$
x_{c}(t)=M \bar{x}_{c}(t),
$$

where the constant matrix $M$ is a block modal matrix of $E_{n}$ and determined by means of the extended matrix sign function $[23,24]$. The $M$ matrix of state space transformation is as follows.

Step 1. Find $\operatorname{sign}\left(\widetilde{E}_{n}\right)$ using the extended matrix sign function with an adequate $\omega$, where

$$
\widetilde{E}_{n}=\left(E_{n}-\omega I_{n}\right)\left(E_{n}+\omega I_{n}\right)^{-1} .
$$

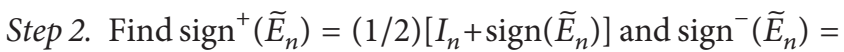
$(1 / 2)\left[I_{n}-\operatorname{sign}\left(\widetilde{E}_{n}\right)\right]$. 
Step 3. Construct the matrix $M=\left[\operatorname{ind}\left(\operatorname{sign}^{+}\left(\widetilde{E}_{n}\right)\right)\right.$ $\left.\operatorname{ind}\left(\operatorname{sign}^{-}\left(\widetilde{E}_{n}\right)\right)\right]$, where $\operatorname{ind}(\cdot)$ represents the collection of linearly independent column vectors of $(\cdot)$.

Substituting (5) into (3) and multiplying by $M^{-1}$ on the left yield

$$
\begin{aligned}
M^{-1} E_{n} M \dot{\bar{x}}_{c}(t)= & M^{-1} A_{n} M \bar{x}_{c}(t)+\sum_{i=1}^{N_{1}} M^{-1} \widehat{A}_{n, i} M \bar{x}_{c}\left(t-\tau_{s, i}\right) \\
& +\sum_{j=1}^{N_{2}} M^{-1} B_{n, j} u_{c}\left(t-\tau_{i, j}\right) \\
= & \frac{1}{\beta}\left(I_{n}-\alpha E_{n}\right) \bar{x}_{c}(t) \\
& +\sum_{i=1}^{N_{1}} M^{-1} \widehat{A}_{n, i} M \bar{x}_{c}\left(t-\tau_{s, i}\right) \\
& +\sum_{j=1}^{N_{2}} M^{-1} B_{n, j} u_{c}\left(t-\tau_{i, j}\right) .
\end{aligned}
$$

If $M^{-1} \widehat{A}_{n, i} M$ can be diagonalized, then (7) yields,

$$
\begin{aligned}
& {\left[\begin{array}{l|l}
\bar{E}_{1} & O \\
\hline & \bar{E}_{2}
\end{array}\right] \dot{\bar{x}}_{c}(t)} \\
& = \\
& \left.\begin{array}{c|c}
\frac{1}{\beta}\left(I_{k}-\alpha \bar{E}_{1}\right) & O \\
\hline O & \frac{1}{\beta}\left(I_{n-\kappa}-\alpha \bar{E}_{2}\right)
\end{array}\right] \bar{x}_{c}(t) \\
& \quad+\sum_{i=1}^{N_{1}}\left[\begin{array}{c|c}
\bar{A}_{1, i} & O \\
\hline O & \bar{A}_{2, i}
\end{array}\right] \bar{x}_{c}\left(t-\tau_{s, i}\right) \\
& \quad+\sum_{j=1}^{N_{2}}\left[\frac{\bar{B}_{1, j}}{\bar{B}_{2, j}}\right] u_{c}\left(t-\tau_{i, j}\right),
\end{aligned}
$$

where $\bar{x}_{c}(t)=\left[\bar{x}_{s}^{T}(t), \bar{x}_{f}^{T}(t)\right]^{T}$ and $M^{-1} E_{n} M=$ block diagonal $\left\{\bar{E}_{1}, \bar{E}_{2}\right\} . \bar{E}_{1}$ is invertible with $\operatorname{rank}\left(\bar{E}_{1}\right)=\operatorname{deg}\{\operatorname{det}$ $\left.\left(s E_{r}-A\right)\right\} \triangleq k,\left[\bar{B}_{1, j}^{T}, \bar{B}_{2, j}^{T}\right]^{T}=M^{-1} B_{n, j}$, and $\bar{E}_{2}$ is a nilpotent matrix with dimension $(n-k) \times(n-k)$. Since $\operatorname{det}\left(I_{n-k}-\alpha \bar{E}_{2}\right)=$ 1 , it is invertible. Simplifying (8) by premultiplying the block diagonal $\left\{\bar{E}_{1}^{-1}, \beta\left(I_{n-k}-\alpha \bar{E}_{2}\right)^{-1}\right\}$ on both sides, one has

$$
\begin{aligned}
{\left[\begin{array}{c|c}
I_{k} & O \\
\hline O & \bar{E}_{f}
\end{array}\right] \dot{\bar{x}}_{c}(t)=} & {\left[\begin{array}{c|c}
\bar{A}_{s} & O \\
\hline O & I_{n-\kappa}
\end{array}\right] \bar{x}_{c}(t) } \\
& +\sum_{i=1}^{N_{1}}\left[\begin{array}{c|c}
\widetilde{A}_{1, i} & O \\
\hline O & \widetilde{A}_{2, i}
\end{array}\right] \bar{x}_{c}\left(t-\tau_{s, i}\right) \\
& +\sum_{j=1}^{N_{2}}\left[\begin{array}{c}
\bar{B}_{s, j} \\
\hline \bar{B}_{f, j}
\end{array}\right] u_{c}\left(t-\tau_{i, j}\right),
\end{aligned}
$$

where

$$
\begin{gathered}
\bar{E}_{f}=\beta\left(I_{n-\kappa}-\alpha \bar{E}_{2}\right)^{-1} \bar{E}_{2}, \\
\bar{A}_{s}=\frac{1}{\beta}\left(\bar{E}_{1}^{-1}-\alpha I_{\kappa}\right),
\end{gathered}
$$

$$
\begin{gathered}
\widetilde{A}_{1, i}=\bar{E}_{1}^{-1} \bar{A}_{1, i}, \\
\widetilde{A}_{2, i}=\beta\left(I_{n-\kappa}-\alpha \bar{E}_{2}\right)^{-1} \bar{A}_{2, i}, \\
\bar{B}_{s, j}=\bar{E}_{1}^{-1} \bar{B}_{1, j}, \\
\bar{B}_{f, j}=\beta\left(I_{n-\kappa}-\alpha \bar{E}_{2}\right)^{-1} \bar{B}_{2, j} .
\end{gathered}
$$

Remarkably, since

$$
\operatorname{rank}(E)-\operatorname{deg}\{\operatorname{det}(s E-A)\}=\operatorname{rank}\left(\bar{E}_{f}\right),
$$

it is much easier to determine the number of the impulsive mode using the above equation relating to (9).

For simplicity, only those singular systems that include at least one impulsive mode are discussed. First, assume that the singular system (9) has $q$; then, $\operatorname{rank}\left(\bar{E}_{f}\right)=q$. By a previously proposed method [12], the preliminary feedback gain $K_{f, j}$ is found and $K_{f, j}$ is proven to eliminate the impulsive modes. For the time-delay singular system (9), the proposed method yields a similar result (Appendix A) to that previously developed method [12] and the linear preliminary feedback control is

$$
\begin{aligned}
u_{c}\left(t-\tau_{i, j}\right) & =-K_{f, j} \hat{x}_{c, f}(t)+v_{c}\left(t-\tau_{i, j}\right) \\
& =-\left[O_{m \times k}, K_{f, j}\right] \widehat{x}_{c}(t)+v_{c}\left(t-\tau_{i, j}\right) .
\end{aligned}
$$

The time-delay singular system (9) can be transformed into (Appendix A)

$$
\begin{aligned}
E_{k} \dot{\hat{x}}_{c}(t)= & A_{k} \widehat{x}_{c}(t)+\sum_{i=1}^{N_{1}} \widehat{A}_{k, i} \widehat{x}_{c}\left(t-\tau_{s, i}\right) \\
& +\sum_{j=1}^{N_{2}} B_{k, j} v_{c}\left(t-\tau_{i, j}\right),
\end{aligned}
$$

where

$$
\begin{aligned}
& E_{k}=\left[\begin{array}{l|l}
I_{k} & O \\
\hline O & \widehat{E}_{f}
\end{array}\right], \\
& A_{k}=\left[\begin{array}{c|c}
\widehat{A}_{s} & -\sum_{j=1}^{N_{2}} \widehat{B}_{s, j} K_{f, j} \\
\hline O & I_{n-k}-\sum_{j=1}^{N_{2}} \widehat{B}_{f, j} K_{f, j}
\end{array}\right], \\
& \widehat{A}_{k, i}=\left[\begin{array}{c|c}
\widetilde{A}_{1, i} & O \\
\hline O & \widetilde{A}_{2, i}
\end{array}\right], \\
& B_{k, j}=\left[\begin{array}{c}
\widehat{B}_{s, j} \\
\hdashline \widehat{B}_{f, j}
\end{array}\right],
\end{aligned}
$$

in which

$$
\begin{gathered}
\widehat{E}_{f}=U^{-1} \bar{E}_{f} U, \\
\widehat{A}_{s}=\bar{A}_{s}, \\
\widetilde{A}_{1, i}=\bar{E}_{1}^{-1} \bar{A}_{1, i},
\end{gathered}
$$




$$
\begin{gathered}
\widetilde{A}_{2, i}=\beta\left(I_{n-\kappa}-\alpha \bar{E}_{2}\right)^{-1} \bar{A}_{2, i}, \\
\widehat{B}_{s, j}=\bar{B}_{s, j}, \\
\widehat{B}_{f, j}=U^{-1} \bar{B}_{f, j},
\end{gathered}
$$

and $U$ is a modal matrix of $\bar{E}_{f}$ with dimension $(n-k) \times$ $(n-k)$ such that $U^{-1} \bar{E}_{f} U$ is in the Jordan block form. The time-delay singular system in (13) is obtained by applying the linear preliminary feedback control law $u(t)$ from (12) to the system that is given by (9). Equation (13) has the $q$ finite modes (where $q=\operatorname{rank}\left(\bar{E}_{f}\right)=\operatorname{rank}\left(\widehat{E}_{f}\right)$ ) and the $k$ original finite modes.All of these finite modes are guaranteed to be controllable. The next task is to decompose the singular system into a reduced-order regular system with $(k+q)$ controllable finite modes and the nondynamic equation with $(n-k-q)$ infinite nondynamic ones. This task can be accomplished by using previously outlined steps. First, the regular form is transformed into a standard one by multiplying (13) by $\left(\gamma E_{k}+\eta A_{k}\right)^{-1}$, where $\gamma$ and $\eta$ are arbitrary scalars such that $\left(\gamma E_{k}+\eta A_{k}\right)$ is invertible. Therefore,

$$
\begin{aligned}
\left(\gamma E_{k}\right. & \left.+\eta A_{k}\right)^{-1} E_{k} \dot{\hat{x}}_{c}(t) \\
= & \left(\gamma E_{k}+\eta A_{k}\right)^{-1} A_{k} \widehat{x}_{c}(t) \\
& +\sum_{i=1}^{N_{1}}\left(\gamma E_{k}+\eta A_{k}\right)^{-1} \widehat{A}_{k, i} \widehat{x}_{c}\left(t-\tau_{s, i}\right) \\
& +\sum_{j=1}^{N_{2}}\left(\gamma E_{k}+\eta A_{k}\right)^{-1} B_{k, j} v_{c}\left(t-\tau_{i, j}\right) .
\end{aligned}
$$

Let

$$
\widehat{x}_{c}(t)=\widetilde{M} \tilde{x}_{c}(t),
$$

where the constant matrix $\widetilde{M}$ is determined by using the extended matrix sign function. The procedure is the same as that elucidated above for finding $M$, except that it operates on $\left(\gamma E_{k}+\eta A_{k}\right)^{-1} E_{k}$. Substituting (17) into (16) and multiplying by $\widetilde{M}^{-1}$ yield

$$
\begin{aligned}
\widetilde{M}^{-1}\left(\gamma E_{k}+\eta A_{k}\right)^{-1} E_{k} \widetilde{M}_{\tilde{x}_{c}}(t) \\
=\widetilde{M}^{-1}\left(\gamma E_{k}+\eta A_{k}\right)^{-1} A_{k} \widetilde{M} \widetilde{x}_{c}(t) \\
\quad+\sum_{i=1}^{N_{1}} \widetilde{M}^{-1}\left(\gamma E_{k}+\eta A_{k}\right)^{-1} \widehat{A}_{k, i} \widetilde{M} \widetilde{x}_{c}\left(t-\tau_{s, i}\right) \\
\quad+\sum_{j=1}^{N_{2}} \widetilde{M}^{-1}\left(\gamma E_{k}+\eta A_{k}\right)^{-1} B_{k, j} v_{c}\left(t-\tau_{i, j}\right) \\
=\widetilde{M}^{-1} \frac{1}{\eta}\left[I_{n}-\gamma\left(\gamma E_{k}+\eta A_{k}\right)^{-1} E_{k}\right] \widetilde{M} \widetilde{x}_{c}(t) \\
\quad+\sum_{i=1}^{N_{1}} \widetilde{M}^{-1}\left(\gamma E_{k}+\eta A_{k}\right)^{-1} \widehat{A}_{k, i} \widetilde{M} \widetilde{x}_{c}\left(t-\tau_{s, i}\right)
\end{aligned}
$$

$$
\begin{aligned}
& +\sum_{j=1}^{N_{2}} \widetilde{M}^{-1}\left(\gamma E_{k}+\eta A_{k}\right)^{-1} B_{k, j} \nu_{c}\left(t-\tau_{i, j}\right) \\
= & \frac{1}{\eta}\left[I_{n}-\gamma \widetilde{M}^{-1}\left(\gamma E_{k}+\eta A_{k}\right)^{-1} E_{k} \widetilde{M}\right] \widetilde{x}_{c}(t) \\
& +\sum_{i=1}^{N_{1}} \widetilde{M}^{-1}\left(\gamma E_{k}+\eta A_{k}\right)^{-1} \widehat{A}_{k, i} \widetilde{M} \widetilde{x}_{c}\left(t-\tau_{s, i}\right) \\
& +\sum_{j=1}^{N_{2}} \widetilde{M}^{-1}\left(\gamma E_{k}+\eta A_{k}\right)^{-1} B_{k, j} \nu_{c}\left(t-\tau_{i, j}\right) .
\end{aligned}
$$

That is,

$$
\begin{aligned}
& {\left[\begin{array}{c|c}
\bar{E}_{s k} & O \\
\hline O & \bar{E}_{f k}
\end{array}\right] \dot{\tilde{x}}_{c}(t)} \\
& =\left[\begin{array}{c|c}
\frac{1}{\eta}\left(I_{k+q}-\gamma \bar{E}_{s k}\right) & O \\
\hline O & \frac{1}{\eta}\left(I_{n-k-q}-\gamma \bar{E}_{f k}\right)
\end{array}\right] \tilde{x}_{c}(t) \\
& +\sum_{i=1}^{N_{1}}\left[\begin{array}{c|c}
\Lambda_{1, i} & O \\
\hline O & \Lambda_{2, i}
\end{array}\right] \tilde{x}_{c}\left(t-\tau_{s, i}\right) \\
& \left.+\sum_{j=1}^{N_{2}}\left[\bar{B}_{s k, j}\right] v_{f k, j}\right] v_{c}\left(t-\tau_{i, j}\right),
\end{aligned}
$$

where $\tilde{x}_{c}(t)=\left[\tilde{x}_{s}^{T}(t), \tilde{x}_{f}^{T}(t)\right]^{T}, \widetilde{M}^{-1}\left(\gamma E_{k}+\eta A_{k}\right)^{-1} E_{k} \widetilde{M}=$ block diagonal $\left\{\bar{E}_{s k}, \bar{E}_{f k}\right\}=$ block diagonal $\left\{\bar{E}_{s k}, O_{(n-q-k)}\right\}$. $\bar{E}_{s k}$ is invertible with $\operatorname{rank}\left(\bar{E}_{s k}\right)=\operatorname{deg}\left\{\operatorname{det}\left(s E_{k}-A_{k}\right)\right\}=(q+k)$. $\bar{E}_{f k}$ is a null matrix and $\left[\bar{B}_{s k, j}^{T}, \bar{B}_{f k, j}^{T}\right]^{T}=\widetilde{M}^{-1}\left(\gamma E_{k}+\eta A_{k}\right)^{-1}$ $B_{k, j}$. In (19), $\widetilde{M}^{-1}\left(\gamma E_{k}+\eta A_{k}\right)^{-1} \widehat{A}_{k, i}$ is assumed to be able to be diagonalized as block diagonal $\left\{\Lambda_{1, i}, \Lambda_{2, i}\right\}$. Then, (19) can be rewritten as

$$
\begin{aligned}
& \dot{\tilde{x}}_{s}(t)= \frac{1}{\eta}\left(\bar{E}_{s k}^{-1}-\gamma I_{k+q}\right) \tilde{x}_{s}(t) \\
&+\sum_{i=1}^{N_{1}} \bar{E}_{s k}^{-1} \Lambda_{1, i} \tilde{x}_{s}\left(t-\tau_{s, i}\right)+\sum_{j=1}^{N_{2}} \bar{E}_{s k}^{-1} \bar{B}_{s k, j} \nu_{c}\left(t-\tau_{i, j}\right), \\
& 0=\tilde{x}_{f}(t)+\sum_{i=1}^{N_{1}} \eta \Lambda_{2, i} \tilde{x}_{f}\left(t-\tau_{s, i}\right)+\sum_{j=1}^{N_{2}} \eta \bar{B}_{f k, j} v_{c}\left(t-\tau_{i, j}\right),
\end{aligned}
$$

and the time-delay singular system output (1b) can be rewritten as (Appendix B)

$$
\begin{aligned}
y_{c}(t) & =C x_{c}\left(t-\tau_{o}\right) \\
& =\left[\begin{array}{ll}
C_{1} & C_{2}
\end{array}\right]\left[\begin{array}{l}
\tilde{x}_{s}\left(t-\tau_{o}\right) \\
\tilde{x}_{f}\left(t-\tau_{o}\right)
\end{array}\right]
\end{aligned}
$$




$$
\begin{aligned}
& =C_{1} \tilde{x}_{s}\left(t-\tau_{o}\right)+C_{2} \tilde{x}_{f}\left(t-\tau_{o}\right) \\
& =C_{1} \tilde{x}_{s}\left(t-\tau_{o}\right)-C_{2} \sum_{j=1}^{N_{2}} \bar{B}_{f k, j} v_{c}\left(t-\tau_{i, j}\right),
\end{aligned}
$$

where $C M V \widetilde{M}=\left[\begin{array}{ll}C_{1} & C_{2}\end{array}\right]$.

Finally, the time-delay singular system (1a) and (1b) can be decomposed as the equivalent regular time-delay system as follows:

$$
\begin{gathered}
\dot{\tilde{x}}_{s}(t)=A_{s} \widetilde{x}_{s}(t)+\sum_{i=1}^{N_{1}} \widehat{A}_{d, i} \widetilde{x}_{s}\left(t-\tau_{s, i}\right)+\sum_{j=1}^{N_{2}} B_{d, j} v_{c}\left(t-\tau_{i, j}\right), \\
y_{c}(t)=C_{1} \widetilde{x}_{s}\left(t-\tau_{o}\right)-\sum_{j=1}^{N_{2}} D_{j} v_{c}\left(t-\tau_{i, j}\right)
\end{gathered}
$$

where

$$
\begin{gathered}
A_{s}=\frac{1}{\eta}\left(\bar{E}_{s k}^{-1}-\gamma I_{k+q}\right), \\
\widehat{A}_{d, i}=\bar{E}_{s k}^{-1} \Lambda_{1, i}, \\
B_{d, j}=\bar{E}_{s k}^{-1} \bar{B}_{s k, j}, \\
D_{j}=C_{2} \bar{B}_{f k, j} .
\end{gathered}
$$

Following the transformation, the time-delay singular system (1a) and (1b) can be converted into a regular system (22a) and (22b) that contains a direct transmission term from input to output and the impulsive mode can be eliminated by means of the method [12]. In the next section, (22a) and (22b) will be used to develop the new optimal tracker and observer for a time-delay singular system (la) and (lb) with a series of time-delays. The proposed approaches are more general and applicable to actual systems.

\subsection{Based on Digital Redesign and Optimal Control to}

Discretize the Continuous Time-Delay Singular System and Construct the Performance Index

3.2.1. Discretization of Continuous Time-Delay Singular System. Consider the continuous time-delay singular system (22a) and (22b). To discretize (22a) and (22b), assume that $v_{c}(t)$ is a piecewise constant input function:

$$
v_{c}(t)=v_{d}(k T), \quad k T \leq t<(k+1) T,
$$

where $T$ is the sampling period. Let the state delay time be given by $\tau_{s, i}=\rho_{i} T+\Gamma_{i}$, where $0 \leq \Gamma_{i}<T$ and $\rho_{i} \geq 0$ is an integer, and let the input delay time be given by $\tau_{i, j}=\eta_{j} T+$ $\sigma_{j}$, where $0 \leq \sigma_{j}<T$ and $\eta_{j} \geq 0$ is an integer. The timedelay singular system (22a) and (22b), by both the Newton extrapolation method and the Chebyshev quadrature method $[25,26]$, becomes

$$
\begin{aligned}
& \tilde{x}_{d s}((k+1) T) \\
& =G \widetilde{x}_{d s}(k T)+\sum_{i=1}^{N_{1}}\left[\widehat{G}_{i}^{(1)} \widetilde{x}_{d s}\left(k T-\rho_{i} T+T\right)\right.
\end{aligned}
$$

$$
\begin{gathered}
+\widehat{G}_{i}^{(2)} \tilde{x}_{d s}\left(k T-\rho_{i} T\right) \\
\left.+\widehat{G}_{i}^{(3)} \widetilde{x}_{d s}\left(k T-\rho_{i} T-T\right)\right] \\
+\sum_{j=1}^{N_{2}}\left[H_{j}^{(0)} v_{d}\left(k T-\eta_{j} T\right)+H_{j}^{(1)} v_{d}\left(k T-\eta_{j} T-T\right)\right],
\end{gathered}
$$

where

$$
\begin{gathered}
G=e^{A_{s} T}, \\
\widehat{G}_{i}^{(1)}=\frac{T}{2}\left[Q_{i}^{(2)}+Q_{i}^{(3)}\right] \widehat{A}_{d, i}, \\
\widehat{G}_{i}^{(2)}=T\left[Q_{i}^{(1)}-Q_{i}^{(3)}\right] \widehat{A}_{d, i}, \\
\widehat{G}_{i}^{(3)}=\frac{T}{2}\left[Q_{i}^{(3)}-Q_{i}^{(2)}\right] \widehat{A}_{d, i}, \\
H_{j}^{(0)}=\left[G^{1-\gamma_{j}}-I_{n}\right] A_{s}^{-1} B_{d, j}, \\
H_{j}^{(1)}=\left[G-G^{1-\gamma_{j}}\right] A_{s}^{-1} B_{d, j},
\end{gathered}
$$

in which

$$
\begin{gathered}
\gamma_{j}=\frac{\sigma_{j}}{T}, \quad \beta_{i}=\frac{\Gamma_{i}}{T}, \\
Q_{i}^{(1)}=\left[G-I_{n}\right]\left(A_{s} T\right)^{-1}, \\
Q_{i}^{(2)}=\left[Q_{i}^{(1)}-\left(1-\beta_{i}\right) I_{n}-\beta_{i} G\right]\left(A_{s} T\right)^{-1}, \\
Q_{i}^{(3)}=\left[2 Q_{i}^{(2)}-\left(1-\beta_{i}\right)^{2} I_{n}-\beta_{i}^{2} G\right]\left(A_{s} T\right)^{-1} .
\end{gathered}
$$

Some terms in (25) may be combined because of the same delay, so (25) can be reduced to

$$
\begin{aligned}
\widetilde{x}_{d s}((k+1) T)= & \bar{G} \widetilde{x}_{d s}(k T)+\sum_{i=1}^{M_{1}} \bar{G}_{i} \widetilde{x}_{d s}(k T-i T) \\
& +\bar{H} v_{d}(k T)+\sum_{j=1}^{M_{2}} \bar{H}_{j} v_{d}(k T-j T) .
\end{aligned}
$$

For the output (22b), the time-delay state $x_{c}\left(t-\tau_{o}\right)$ for $k T \leq$ $t-\tau_{o}<(k+1) T$ must be evaluated. System (22a) and (22b) can be rewritten as

$$
\begin{aligned}
\widetilde{x}_{s}\left(t-\tau_{o}\right)= & e^{A_{s}\left(t-\tau_{o}-k T\right)} \tilde{x}_{d s}(k T) \\
& +\sum_{i=1}^{N_{1}} \int_{k T}^{t-\tau_{o}} e^{A_{s}\left(t-\tau_{o}-\lambda\right)} \widehat{A}_{d, i} \tilde{x}_{d s}\left(\lambda-\tau_{s, i}\right) d \lambda \\
& +\sum_{j=1}^{N_{2}} \int_{k T}^{t-\tau_{o}} e^{A_{s}\left(t-\tau_{o}-\lambda\right)} B_{d, j} v_{d}\left(\lambda-\tau_{i, j}\right) d \lambda \\
= & \delta_{1}\left(t-\tau_{o}-k T\right) \tilde{x}_{d s}(k T) \\
& \quad+\sum_{i=1}^{N_{1}}\left[\widehat{\delta}_{i}^{(1)}\left(t-\tau_{o}-k T\right) \tilde{x}_{d s}\left(k T-\rho_{i} T+T\right)\right.
\end{aligned}
$$




$$
\begin{aligned}
&+\widehat{\delta}_{i}^{(2)}\left(t-\tau_{o}-k T\right) \tilde{x}_{d s}\left(k T-\rho_{i} T\right) \\
&+\widehat{\delta}_{i}^{(3)}\left(t-\tau_{o}-k T\right) \\
&\left.\times \widetilde{x}_{d s}\left(k T-\rho_{i} T-T\right)\right] \\
&+\sum_{j=1}^{N_{2}}\left[\varphi_{j}^{(0)}\left(t-\tau_{o}-k T\right) v_{d}\left(k T-\eta_{j} T\right)\right. \\
& \quad+\varphi_{j}^{(1)}\left(t-\tau_{o}-k T\right) \\
&\left.\quad \times v_{d}\left(k T-\eta_{j} T-T\right)\right],
\end{aligned}
$$

where

$$
\begin{gathered}
\delta_{1}\left(t-\tau_{o}-k T\right)=e^{A_{s}\left(t-\tau_{o}-k T\right)}, \\
\widehat{\delta}_{i}^{(1)}\left(t-\tau_{o}-k T\right)=\frac{T}{2}\left[q_{i}^{(2)}+q_{i}^{(3)}\right] \widehat{A}_{d, i}, \\
\widehat{\delta}_{i}^{(2)}\left(t-\tau_{o}-k T\right)=T\left[q_{i}^{(1)}-q_{i}^{(3)}\right] \widehat{A}_{d, i}, \\
\widehat{\delta}_{i}^{(3)}\left(t-\tau_{o}-k T\right)=\frac{T}{2}\left[q_{i}^{(3)}-q_{i}^{(2)}\right] \widehat{A}_{d, i}, \\
\varphi_{j}^{(0)}\left(t-\tau_{o}-k T\right) \\
= \begin{cases}O_{n \times m}, & t-\tau_{o}<\sigma_{j}, \\
{\left[e^{-A_{s} \sigma_{j}} \delta_{1}\left(t-\tau_{o}-k T\right)-I_{n}\right] A_{s}^{-1} B_{d, j},} & t-\tau_{o} \geq \sigma_{j},\end{cases} \\
\varphi_{j}^{(1)}\left(t-\tau_{o}-k T\right) \\
= \begin{cases}{\left[\delta_{1}\left(t-\tau_{o}-k T\right)-I_{n}\right] A_{s}^{-1} B_{d, j},} & t-\tau_{o}<\sigma_{j} \\
\delta_{1}\left(t-\tau_{o}-k T\right)\left[I_{n}-e^{-A_{s} \sigma_{j}}\right] A_{s}^{-1} B_{d, j}, & t-\tau_{o} \geq \sigma_{j},\end{cases}
\end{gathered}
$$

in which

$$
\begin{aligned}
q_{i}^{(1)}= & {\left[\delta_{1}\left(t-\tau_{o}-k T\right)-I_{n}\right]\left(A_{s} T\right)^{-1}, } \\
q_{i}^{(2)}= & {\left[q_{i}^{(1)}-\left(\frac{t-\tau_{o}-k T}{T}-\beta_{i}\right) I_{n}\right.} \\
& \left.-\beta_{i} \delta_{1}\left(t-\tau_{o}-k T\right)\right]\left(A_{s} T\right)^{-1}, \\
q_{i}^{(3)}= & {\left[2 q_{i}^{(2)}-\left(\frac{t-\tau_{o}-k T}{T}-\beta_{i}\right)^{2} I_{n}\right.} \\
& \left.+\beta_{i}^{2} \delta_{1}\left(t-\tau_{o}-k T\right)\right]\left(A_{s} T\right)^{-1} .
\end{aligned}
$$

Also, some terms in (29) may be combined as in (28), and (29) may be rewritten as

$$
\begin{aligned}
\tilde{x}_{s}\left(t-\tau_{o}\right)= & \bar{\delta}_{0}\left(t-\tau_{o}-k T\right) \tilde{x}_{d s}(k T) \\
& +\sum_{i=1}^{M_{1}} \bar{\delta}_{i}\left(t-\tau_{o}-k T\right) \tilde{x}_{d s}(k T-i T)
\end{aligned}
$$

$$
\begin{gathered}
+\bar{\varphi}_{0}\left(t-\tau_{o}-k T\right) v_{d}(k T) \\
+\sum_{j=1}^{M_{2}} \bar{\varphi}_{j}\left(t-\tau_{o}-k T\right) v_{d}(k T-j T) .
\end{gathered}
$$

Then, the output (22b) can be rewritten as

$$
\begin{aligned}
y_{c}(t)= & C_{1} \tilde{x}_{s}\left(t-\tau_{o}\right)-\sum_{j=1}^{N_{2}} D_{j} v_{c}\left(t-\tau_{i, j}\right) \\
= & C_{1} \bar{\delta}_{0}\left(t-\tau_{o}-k T\right) \tilde{x}_{d s}(k T) \\
& +\sum_{i=1}^{M_{1}} C_{1} \bar{\delta}_{i}\left(t-\tau_{o}-k T\right) \tilde{x}_{d s}(k T-i T) \\
& +C_{1} \bar{\varphi}_{0}\left(t-\tau_{o}-k T\right) v_{d}(k T) \\
& +\sum_{j=1}^{M_{2}} C_{1} \bar{\varphi}_{j}\left(t-\tau_{o}-k T\right) v_{d}(k T-j T) \\
& -\sum_{j=1}^{N_{2}}\left[D_{j}^{(0)} v_{d}\left(k T-\eta_{j} T\right)\right. \\
& \left.+D_{j}^{(1)} v_{d}\left(k T-\eta_{j} T-T\right)\right]
\end{aligned}
$$

where

$$
\begin{gathered}
D_{j}^{(0)}=D_{j}^{*}\left(B_{d, j}^{T} B_{d, j}\right)^{-1} H_{j}^{(0)}, \\
D_{j}^{(1)}=D_{j}^{*}\left(B_{d, j}^{T} B_{d, j}\right)^{-1} H_{j}^{(1)}, \\
D_{j}^{*}=\left[\begin{array}{ll}
D_{j} & O
\end{array}\right]^{T} .
\end{gathered}
$$

Similarly, some terms in (33) can be combined, so (33) can be rewritten as

$$
\begin{aligned}
y_{c}(t)= & C_{1} \bar{\delta}_{0}\left(t-\tau_{o}-k T\right) \tilde{x}_{d s}(k T) \\
& +\sum_{i=1}^{M_{1}} C_{1} \bar{\delta}_{i}\left(t-\tau_{o}-k T\right) \tilde{x}_{d s}(k T-i T) \\
& \quad+C_{1} \bar{\varphi}_{0}^{*}\left(t-\tau_{o}-k T\right) v_{d}(k T) \\
& +\sum_{j=1}^{M_{2}} C_{1} \bar{\varphi}_{j}^{*}\left(t-\tau_{o}-k T\right) v_{d}(k T-j T) .
\end{aligned}
$$

Thus, the discretization of continuous time-delay singular system (22a) and (22b) is carried out using (28) and (35).

3.2.2. Establishing Performance Index for Discrete Time-Delay Singular System. The optimal state-feedback control law minimizes the following performance cost function:

$$
J=\int_{0}^{t_{f}}\left\{\left[C_{1} \tilde{x}_{s}\left(t-\tau_{o}\right)-\sum_{j=1}^{N_{2}} D_{j} v_{c}\left(t-\tau_{i, j}\right)-r(t)\right]^{T}\right.
$$




$$
\begin{aligned}
& \times Q\left[C_{1} \tilde{x}_{s}\left(t-\tau_{o}\right)-\sum_{j=1}^{N_{2}} D_{j} v_{c}\left(t-\tau_{i, j}\right)-r(t)\right] \\
& \left.+v_{c}^{T}(t) R v_{c}(t)\right\},
\end{aligned}
$$

where $Q$ is the positive semidefinite matrix, $R$ is the positive definite matrix, $r(t) \in \mathfrak{R}^{q}$ is the reference input vector, and the final time $t_{f}<\infty$. To discretize the cost function $J$, given by (36), $t_{f}=N T$ is chosen and $J$ can be rewritten as

$$
J=\sum_{k=0}^{N-1} \int_{k T}^{(k+1) T}\left\{\left[C_{1} \widetilde{x}_{s}\left(t-\tau_{o}\right)\right.\right.
$$

$$
\begin{gathered}
\left.-\sum_{j=1}^{N_{2}} D_{j} v_{c}\left(t-\tau_{i, j}\right)-r(t)\right]^{T} \\
\times Q\left[C_{1} \tilde{x}_{s}\left(t-\tau_{o}\right)\right. \\
\left.\quad-\sum_{j=1}^{N_{2}} D_{j} v_{c}\left(t-\tau_{i, j}\right)-r(t)\right] \\
\left.+v_{c}^{T}(t) R v_{c}(t)\right\} .
\end{gathered}
$$

Let $r^{*}(k T) \in \Re^{q}$ be the piecewise-constant reference input vector to be determined in terms of $r(k T)$ for the tracking purpose. Then, cost function (37) can be rewritten as [27]

$$
\begin{gathered}
J=\sum_{k=0}^{N-1} \int_{k T}^{(k+1) T}\left\{\left[\bar{\delta}_{0}\left(t-\tau_{o}-k T\right) \tilde{x}_{d s}(k T)+\sum_{i=1}^{M_{1}} \bar{\delta}_{i}\left(t-\tau_{o}-k T\right) \tilde{x}_{d s}(k T-i T)+\bar{\varphi}_{0}^{*}\left(t-\tau_{o}-k T\right) v_{d}(k T)\right.\right. \\
\left.+\sum_{j=1}^{M_{2}} \bar{\varphi}_{j}^{*}\left(t-\tau_{o}-k T\right) v_{d}(k T-j T)-r^{*}(k T)\right]^{T} \\
\times Q\left[\bar{\delta}_{0}\left(t-\tau_{o}-k T\right) \tilde{x}_{d s}(k T)+\sum_{i=1}^{M_{1}} \bar{\delta}_{i}\left(t-\tau_{o}-k T\right) \tilde{x}_{d s}(k T-i T)+\bar{\varphi}_{0}^{*}\left(t-\tau_{o}-k T\right) v_{d}(k T)\right. \\
\left.\left.+\sum_{j=1}^{M_{2}} \bar{\varphi}_{j}^{*}\left(t-\tau_{o}-k T\right) v_{d}(k T-j T)-r^{*}(k T)\right]+v_{d}^{T}(k T) R v_{d}(k T) d t\right\}
\end{gathered}
$$

$=\cdots$

$=\sum_{k=0}^{N-1}\left[\begin{array}{lllllllll}\tilde{x}_{d s}^{T}(k T) & \tilde{x}_{d s}^{T}(k T-T) & \cdots & \tilde{x}_{d s}^{T}\left(k T-M_{1} T\right) & v_{d}^{T}(k T-T) & \cdots & v_{d}^{T}\left(k T-M_{2} T\right) & r^{* T}(k T) & v_{d}^{T}(k T)\end{array}\right]$

$\times\left[\begin{array}{ccccccccc}Q_{1} & Q_{31} & \cdots & Q_{3 M_{1}} & M_{21} & \cdots & M_{2 M_{2}} & -M_{3} & M_{1} \\ Q_{31}^{T} & Q_{211} & \cdots & Q_{21 M_{1}} & M_{511} & \cdots & M_{51 M_{2}} & -M_{61} & M_{41} \\ \vdots & \vdots & \ddots & \vdots & \vdots & \ddots & \vdots & \vdots & \vdots \\ Q_{3 M_{1}}^{T} & Q_{2 M_{1} 1} & \cdots & Q_{2 M_{1} M_{1}} & M_{5 M_{1} 1} & \cdots & M_{5 M_{1} M_{2}} & -M_{6 M_{1}} & M_{4 M_{1}} \\ M_{21}^{T} & M_{511}^{T} & \cdots & M_{5 M_{1} 1}^{T} & R_{211} & \cdots & R_{21 M_{2}} & -M_{81} & R_{41} \\ \vdots & \vdots & \ddots & \vdots & \vdots & \ddots & \vdots & \cdots & \vdots \\ M_{2 M_{2}}^{T} & M_{51 M_{2}}^{T} & \cdots & M_{5 M_{1} M_{2}}^{T} & R_{2 M_{2}} & \cdots & R_{2 M_{2} M_{2}} & -M_{8 M_{2}} & R_{4 M_{2}} \\ -M_{3}^{T} & -M_{61}^{T} & \cdots & -M_{6 M_{1}}^{T} & -M_{81}^{T} & \cdots & -M_{8 M_{2}}^{T} & R_{3} & -M_{7} \\ M_{1}^{T} & M_{41}^{T} & \cdots & M_{4 M_{1}}^{T} & R_{41}^{T} & \cdots & R_{4 M_{2}}^{T} & -M_{7}^{T} & R_{1}\end{array}\right]$

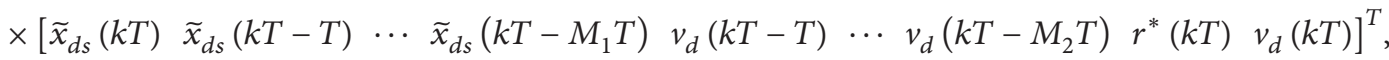


where

$$
\begin{aligned}
& Q_{1}=\int_{k T}^{(k+1) T}\left[\left(C_{1} \bar{\delta}_{0}\left(t-\tau_{o}-k T\right)\right)^{T}\right. \\
& \left.\times Q\left(C_{1} \bar{\delta}_{0}\left(t-\tau_{o}-k T\right)\right)\right] d t, \\
& Q_{2 i j}=\int_{k T}^{(k+1) T}\left[\left(C_{1} \bar{\delta}_{i}\left(t-\tau_{o}-k T\right)\right)^{T}\right. \\
& \left.\times Q\left(C_{1} \bar{\delta}_{j}\left(t-\tau_{o}-k T\right)\right)\right] d t, \\
& Q_{3 i}=\int_{k T}^{(k+1) T}\left[\left(C_{1} \bar{\delta}_{0}\left(t-\tau_{o}-k T\right)\right)^{T}\right. \\
& \left.\times Q\left(C_{1} \bar{\delta}_{i}\left(t-\tau_{o}-k T\right)\right)\right] d t, \\
& M_{1}=\int_{k T}^{(k+1) T}\left[\left(C_{1} \bar{\delta}_{0}\left(t-\tau_{o}-k T\right)\right)^{T}\right. \\
& \left.\times Q\left(C_{1} \bar{\varphi}_{0}^{*}\left(t-\tau_{o}-k T\right)\right)\right] d t, \\
& M_{2 j}=\int_{k T}^{(k+1) T}\left[\left(C_{1} \bar{\delta}_{0}\left(t-\tau_{o}-k T\right)\right)^{T}\right. \\
& \left.\times Q\left(C_{1} \bar{\varphi}_{j}^{*}\left(t-\tau_{o}-k T\right)\right)\right] d t, \\
& M_{3}=\int_{k T}^{(k+1) T}\left[\left(C_{1} \bar{\delta}_{0}\left(t-\tau_{o}-k T\right)\right)^{T} Q\right] d t, \\
& M_{4 i}=\int_{k T}^{(k+1) T}\left[\left(C_{1} \bar{\delta}_{i}\left(t-\tau_{o}-k T\right)\right)^{T}\right. \\
& \left.\times Q\left(C_{1} \bar{\varphi}_{0}^{*}\left(t-\tau_{o}-k T\right)\right)\right] d t,
\end{aligned}
$$

$$
\begin{aligned}
M_{5 i j}=\int_{k T}^{(k+1) T}[ & \left(C_{1} \bar{\delta}_{i}\left(t-\tau_{o}-k T\right)\right)^{T} \\
& \left.\times Q\left(C_{1} \bar{\varphi}_{j}^{*}\left(t-\tau_{o}-k T\right)\right)\right] d t,
\end{aligned}
$$$$
M_{6 i}=\int_{k T}^{(k+1) T}\left[\left(C_{1} \bar{\delta}_{i}\left(t-\tau_{o}-k T\right)\right)^{T} Q\right] d t,
$$$$
M_{7}=\int_{k T}^{(k+1) T}\left[Q\left(C_{1} \bar{\varphi}_{0}^{*}\left(t-\tau_{o}-k T\right)\right)\right] d t,
$$$$
M_{8 j}=\int_{k T}^{(k+1) T}\left[\left(C_{1} \bar{\varphi}_{j}^{*}\left(t-\tau_{o}-k T\right)\right)^{T} Q\right] d t,
$$$$
R_{1}=\int_{k T}^{(k+1) T}\left[\left(C_{1} \bar{\varphi}_{0}^{*}\left(t-\tau_{o}-k T\right)\right)^{T}\right.
$$$$
\left.\times Q\left(C_{1} \bar{\varphi}_{0}^{*}\left(t-\tau_{o}-k T\right)\right)+R\right] d t,
$$$$
R_{2 i j}=\int_{k T}^{(k+1) T}\left[\left(C_{1} \bar{\varphi}_{i}^{*}\left(t-\tau_{o}-k T\right)\right)^{T}\right.
$$$$
\left.\times Q\left(C_{1} \bar{\varphi}_{j}^{*}\left(t-\tau_{o}-k T\right)\right)\right] d t,
$$$$
R_{3}=\int_{k T}^{(k+1) T} Q d t,
$$$$
R_{4 j}=\int_{k T}^{(k+1) T}\left[\left(C_{1} \bar{\varphi}_{i}^{*}\left(t-\tau_{o}-k T\right)\right)^{T}\right.
$$$$
\left.\times Q\left(C_{1} \bar{\varphi}_{j}^{*}\left(t-\tau_{o}-k T\right)\right)\right] d t .
$$

Construct an extended virtual state vector:

$$
X_{d}(k T)=\left[\begin{array}{llllllll}
\tilde{x}_{d s}(k T) & \tilde{x}_{d s}(k T-T) & \cdots & \tilde{x}_{d s}\left(k T-M_{1} T\right) & v_{d}(k T-T) & \cdots & v_{d}\left(k T-M_{2} T\right) & r^{*}(k T)
\end{array}\right]^{T} .
$$

The extended delay-free system that is equivalent to the original time-delay singular system (28) and (35) is obtained as

$$
\begin{gathered}
X_{d}((k+1) T)=\widehat{G}_{e} X_{d}(k T)+\widehat{H}_{e} v_{d}(k T), \\
y_{d}(k T)=\widehat{C}_{e} X_{d}(k T) .
\end{gathered}
$$

We assume that the reference input $r(t)$ is a step function with a constant magnitude, $r^{*}((k+1) T)=r^{*}(k T)$. Designing a system based on such a reference input can lead to predictable time-response characteristics. Although our design methodology is based on a step function, it should be pointed out that the resulting control system, if properly designed, enables to give good time responses for any arbitrary reference input $r(t)$. Also, the reference input $r^{*}(k T)$ is entered in the last row of $X_{d}(k T)$ at the beginning of step $k$. As a result, the extended new system does not have any time-delay terms and it can be utilized to simplify the representation of the cost function (38). Now, (38) can be rewritten as

$$
J=\sum_{k=0}^{N-1}\left[X_{d}^{T}(k T) \quad v_{d}^{T}(k T)\right]\left[\begin{array}{cc}
\widehat{Q} & \widehat{M} \\
\widehat{M}^{T} & \widehat{R}
\end{array}\right]\left[\begin{array}{c}
X_{d}(k T) \\
v_{d}(k T)
\end{array}\right],
$$

where

$$
\begin{aligned}
& {\left[\begin{array}{cccccccc}
Q_{1} & Q_{31} & \cdots & Q_{3 M_{1}} & M_{21} & \cdots & M_{2 M_{2}} & -M_{3} \\
Q_{31}^{T} & Q_{211} & \cdots & Q_{21 M_{1}} & M_{511} & \cdots & M_{51 M_{2}} & -M_{61} \\
\vdots & \vdots & \ddots & \vdots & \vdots & \ddots & \vdots & \vdots \\
Q_{3 M_{1}}^{T} & Q_{2 M_{1} 1} & \cdots & Q_{2 M_{1} M_{1}} & M_{5 M_{1} 1} & \cdots & M_{5 M_{1} M_{2}} & -M_{6 M_{1}} \\
M_{21}^{T} & M_{511}^{T} & \cdots & M_{5 M_{1} 1}^{T} & R_{211} & \cdots & R_{21 M_{2}} & -M_{81} \\
\vdots & \vdots & \ddots & \vdots & \vdots & \ddots & \vdots & \vdots \\
M_{2 M_{2}}^{T} & M_{51 M_{2}}^{T} & \cdots & M_{5 M_{1} M_{2}}^{T} & R_{2 M_{2} 1} & \cdots & R_{2 M_{2} M_{2}} & -M_{8 M_{2}} \\
-M_{3}^{T} & -M_{61}^{T} & \cdots & -M_{6 M_{1}}^{T} & -M_{81}^{T} & \cdots & -M_{8 M_{2}}^{T} & R_{3}
\end{array}\right],} \\
& \widehat{M}=\left[\begin{array}{llllllll}
M_{1}^{T} & M_{41}^{T} & \cdots & M_{4 M_{1}}^{T} & R_{41}^{T} & \cdots & R_{4 M_{2}}^{T} & -M_{7}^{T}
\end{array}\right]^{T}, \\
& \widehat{R}=\left[R_{1}\right] .
\end{aligned}
$$


Then, define a new virtual weighting matrix

$$
\widetilde{Q}=\widehat{Q}-\widehat{M} \widehat{R}^{-1} \widehat{M}^{T}
$$

and a new virtual control input

$$
S(k T)=\widehat{R}^{-1} \widehat{M}^{T} X_{d}(k T)+v_{d}(k T) .
$$

Substituting (44) and (45) into (42) results in a decoupled performance index:

$$
J=\sum_{k=0}^{N-1}\left[X_{d}^{T}(k T) \widetilde{Q} X_{d}(k T)+S^{T}(k T) \widehat{R} S(k T)\right] .
$$

Substituting (45) into the extended delay-free singular system (41a) and (41b) yields

$$
\begin{aligned}
X_{d} & ((k+1) T) \\
& =\widehat{G}_{e} X_{d}(k T)+\widehat{H}_{e} v_{d}(k T) \\
& =\widehat{G}_{e} X_{d}(k T)+\widehat{H}_{e}\left[S(k T)-\widehat{R}^{-1} \widehat{M}^{T} X_{d}(k T)\right] \\
& =\widetilde{G}_{e} X_{d}(k T)+\widehat{H}_{e} S(k T),
\end{aligned}
$$

where $\widetilde{G}_{e}=\widehat{G}_{e}-\widehat{H}_{e} \widehat{R}^{-1} \widehat{M}^{T}$.

Notably, the quadratic optimal control of the system that is given by (41a) and (41b) with the performance index that is given by (42) is equivalent to the quadratic optimal control of the system that is given by (47) with the performance index that is given by (46). The development of the desired optimal virtual control vector $S(k T)$ that minimizes the performance index that is given by (46) can be described as follows.

\subsection{Development of Optimal Tracker for Time-Delay Singular} System with States Available. Let the Hamilton function depend on the cost function (46) [28]:

$$
\begin{aligned}
H_{f}(k T)= & {\left[X_{d}^{T}(k T) \widetilde{Q} X_{d}(k T)+S^{T}(k T) \widehat{R} S(k T)\right] } \\
& +\lambda^{T}((k+1) T)\left[\widetilde{G}_{e} X_{d}(k T)+\widehat{H}_{e} S(k T)\right],
\end{aligned}
$$

where $\lambda(k T)$ is a costate (Lagrange multiplier). Based on the well-developed optimal control theory $[29,30]$, the state equation is

$$
X_{d}((k+1) T)=\widetilde{G}_{e} X_{d}(k T)+\widehat{H}_{e} S(k T),
$$

and the costate equation is

$$
\lambda(k T)=\widetilde{G}_{e}^{T} \lambda((k+1) T)+\widetilde{Q} X_{d}(k T)
$$

with the stationary condition

$$
0=\widehat{H}_{e}^{T} \lambda((k+1) T)+\widehat{R} S(k T),
$$

or

$$
S(k T)=-\widehat{R}^{-1} \widehat{H}_{e}^{T} \lambda((k+1) T),
$$

and the boundary condition is

$$
\lambda(N T)=\widetilde{Q} X_{d}(N T) .
$$

Assume that $\lambda(k T)$ can be written as follows:

$$
\lambda(k T)=P(k T) X_{d}(k T),
$$

where $P(k T)$ is a real symmetric matrix of appropriate dimension. So far, the original optimal tracking problem has been transformed into an optimal regulator problem.

To derive the optimal regulator, (53) is substituted into $(50)$

$$
\begin{aligned}
& P(k T) X_{d}(k T) \\
& \quad=\widetilde{G}_{e}^{T} P((k+1) T) X_{d}((k+1) T)+\widetilde{Q} X_{d}(k T),
\end{aligned}
$$

and (52a), (52b), and (53) are substituted into (49):

$$
\begin{aligned}
X_{d}((k+1) T)= & \widetilde{G}_{e} X_{d}(k T)+\widehat{H}_{e} \widehat{R}^{-1} \widehat{H}_{e}^{T} \lambda((k+1) T) \\
= & \widetilde{G}_{e} X_{d}(k T)+\widehat{H}_{e} \widehat{R}^{-1} \widehat{H}_{e}^{T} P((k+1) T) \\
& \times X_{d}((k+1) T)
\end{aligned}
$$

or

$$
X_{d}((k+1) T)=\left[I+\widehat{H}_{e} \widehat{R}^{-1} \widehat{H}_{e}^{T} P((k+1) T)\right]^{-1} \widetilde{G}_{e} X_{d}(k T) .
$$

Also, substituting (56) into (54) yields

$$
\begin{aligned}
P(k T) X_{d}(k T)= & \widetilde{G}_{e}^{T} P((k+1) T) \\
& \times\left[I+\widehat{H}_{e} \widehat{R}^{-1} \widehat{H}_{e}^{T} P((k+1) T)\right]^{-1} \\
& \times \widetilde{G}_{e} X_{d}(k T)+\widetilde{Q} X_{d}(k T)
\end{aligned}
$$

or

$$
\begin{aligned}
& \left\{P(k T)-\widetilde{G}_{e}^{T} P((k+1) T)\right. \\
& \left.\quad \times\left[I+\widehat{H}_{e} \widehat{R}^{-1} \widehat{H}_{e}^{T} P((k+1) T)\right]^{-1} \widetilde{G}_{e}-\widetilde{Q}\right\} X_{d}(k T)=0 .
\end{aligned}
$$

The above equation must hold for all $X_{d}(k T)$. Hence,

$$
\begin{aligned}
P(k T)= & \widetilde{Q}+\widetilde{G}_{e}^{T} P((k+1) T) \\
& \times\left[I+\widehat{H}_{e} \widehat{R}^{-1} \widehat{H}_{e}^{T} P((k+1) T)\right]^{-1} \widetilde{G}_{e} .
\end{aligned}
$$

Equation (59) is called the Riccati equation. With reference to $(52 \mathrm{a}),(52 \mathrm{~b})$, and (53), when at $k=N$,

$$
\lambda(N T)=\widetilde{Q} X_{d}(N T)=P(N T) X_{d}(N T)
$$

or

$$
P(N T)=\widetilde{Q}
$$


From (59) and (61), all $P(k T)$ for $0 \leq k \leq N$ can be obtained. With reference to (53) and (56), the desired optimal virtual control input that is given by (52a) now becomes

$$
\begin{aligned}
S(k T)= & -\widehat{R}^{-1} \widehat{H}_{e}^{T} \lambda((k+1) T) \\
= & -\widehat{R}^{-1} \widehat{H}_{e}^{T} P((k+1) T) X_{d}((k+1) T) \\
= & -\widehat{R}^{-1} \widehat{H}_{e}^{T} P((k+1) T) \\
& \times\left[I+\widehat{H}_{e} \widehat{R}^{-1} \widehat{H}_{e}^{T} P((k+1) T)\right]^{-1} \widetilde{G}_{e} X_{d}(k T) \\
= & -\widehat{R}^{-1} \widehat{H}_{e}^{T}\left[P^{-1}((k+1) T)+\widehat{H}_{e} \widehat{R}^{-1} \widehat{H}_{e}^{T}\right]^{-1} \\
& \times \widetilde{G}_{e} X_{d}(k T) \\
= & -K(k T) X_{d}(k T),
\end{aligned}
$$

where $K(k T)=\widehat{R}^{-1} \widehat{H}_{e}^{T}\left[P^{-1}((k+1) T)+\widehat{H}_{e} \widehat{R}^{-1} \widehat{H}_{e}^{T}\right]^{-1} \widetilde{G}_{e}$. From (45), the original optimal controller $v_{d}(k T)$ is obtained as follows:

$$
v_{d}(k T)=S(k T)-\widehat{R}^{-1} \widehat{M}^{T} X_{d}(k T)=-\widehat{K}(k T) X_{d}(k T),
$$

where $\widehat{K}(k T)=K(k T)+\widehat{R}^{-1} \widehat{M}^{T}$. Notice that if there are no state and input time delays, the above development can be reduced to that in [30]. Equation (63) can be represented in the following form:

$$
\begin{aligned}
v_{d}(k T) & \\
= & -\widehat{K}(k T) X_{d}(k T) \\
= & -K_{d}(k T) \\
& \times\left[\begin{array}{lllll}
\tilde{x}_{d s}^{T}(k T) & \tilde{x}_{d s}^{T}(k T-T) & \cdots & \tilde{x}_{d s}^{T}\left(k T-M_{1} T\right)
\end{array}\right]^{T} \\
& -F_{d}(k T)\left[\begin{array}{llll}
v_{d}^{T}(k T-T) & \cdots & v_{d}^{T}\left(k T-M_{2} T\right)
\end{array}\right]^{T} \\
& -E_{d}(k T) r^{*}(k T),
\end{aligned}
$$

where $\widehat{K}(k T)=\left[\begin{array}{lll}K_{d}(k T) & F_{d}(k T) & E_{d}(k T)\end{array}\right]$, in which

$$
\begin{gathered}
K_{d}(k T)=\left[\begin{array}{lllll}
K_{d}^{(0)}(k T) & K_{d}^{(1)}(k T) & \cdots & K_{d}^{\left(M_{1}\right)}(k T)
\end{array}\right], \\
F_{d}(k T)=\left[\begin{array}{lllll}
F_{d}^{(1)}(k T) & \cdots & F_{d}^{\left(M_{2}\right)}(k T)
\end{array}\right] .
\end{gathered}
$$

Here, the discrete optimal controller (64) for the continuous time-delay system (22a) and (22b) has been completely derived. Figure 1 presents the realization of the time-varying piecewise-constant optimal controller (64) for the digitally controlled continuous time-delay singular system.

3.4. Development of Observer-Based Suboptimal Tracker for Time-Delay Singular System with States Unavailable. When the states of a continuous time-delay system (22a) and (22b) are not available for measurement, the continuous-time states can be constructed using the recently developed continuous time-delay observers [28, 31, 32]. However, the developed digital tracker (64) requires the extended discrete-time state $\mathrm{X}_{d}(k T)$ in (41a) and (41b). Using the prediction-based digital redesign [27], a new observer-based suboptimal tracker for the time-delay singular system can be designed as follows.

According to the digitally redesigned observer [27] and controller [27], the extended digitally redesigned observer and controller can be represented as

$$
\begin{aligned}
\widehat{X}_{d}((k+1) T)= & \widehat{G}_{o} \widehat{X}_{d}(k T)+\widehat{H}_{o} v_{d}(k T) \\
& +L_{d}\left[y_{d}(k T)-\widehat{C}_{e} \widehat{X}_{d}(k T)\right], \\
v_{d}(k T) & =-\widehat{K}(k T) \widehat{X}_{d}(k T),
\end{aligned}
$$

where $\widehat{\mathrm{X}}_{d}(k T) \in \mathfrak{R}^{p}$ is the estimate of the extended state $\mathrm{X}_{d}(k T) \in \Re^{p}$ in (41a) and (41b),

$$
\begin{aligned}
\widehat{G}_{o} & =\widehat{G}_{e}-L_{d} \widehat{C}_{e} \widehat{G}_{e}, \\
\widehat{H}_{o} & =\widehat{H}_{e}-L_{d} \widehat{C}_{e} \widehat{H}_{e} .
\end{aligned}
$$

Additionally, $\widehat{G}_{e}=e^{\widehat{A} T}$ and $\widehat{H}_{e}=\left[\widehat{G}_{e}-I_{p}\right] \widehat{A}^{-1} \widehat{B}$, where $\widehat{A}=(1 / T) \ln \left(\widehat{G}_{e}\right)$ and $\widehat{B}=\widehat{A}\left(\widehat{G}_{e}-I\right)^{-1} \widehat{H}_{e}$. To determine the extended observer gain $L_{d}$ in (66a), the equivalent extended continuous-time observer (41a) and (41b) and controller (64) can be represented [19], on the basis of the analog observer and controller, as

$$
\begin{gathered}
\dot{\widehat{X}}_{c}(t)=\widehat{A} \widehat{X}_{c}(t)+\widehat{B} v_{c}(t)+L_{c}\left[y_{c}(t)-\widehat{C}_{e} \widehat{X}_{c}(t)\right], \\
v_{c}(t)=-\widehat{K}_{c} \widehat{X}_{c}(t) .
\end{gathered}
$$

The algorithm for computing the analog system matrix $\widehat{A}$ in (67a) from the digital system matrix $\widehat{G}_{e}$ in (41a) via the geometric-series method [15] is as follows:

$$
\begin{aligned}
\widehat{A} & =\frac{1}{T} \ln \left(\widehat{G}_{e}\right) \\
& =\frac{2}{T}\left\{\widehat{R}+\frac{1}{3} \widehat{R}^{3}+\cdots+\frac{1}{n} \widehat{R}^{n}\left[I_{p}-\frac{1}{(1+2 / n)} \widehat{R}^{2}\right]^{-1}\right\} \\
& \quad \text { for }\left|\sigma\left(\widehat{R}^{2}\right)\right|<\left(1+\frac{2}{n}\right) \\
& \cong \frac{2}{T} \widehat{R}\left[I_{p}-\frac{1}{3} \widehat{R}^{2}\right]^{-1} \quad \text { for } n=1 \\
& \cong \frac{2}{T} \widehat{R}\left[I_{p}-\frac{4}{15} \widehat{R}^{2}\right]\left[I_{p}-\frac{3}{5} \widehat{R}^{2}\right]^{-1} \quad \text { for } n=3 \\
& \cong \cdots,
\end{aligned}
$$

where $\widehat{R}=\left[\widehat{G}_{e}-I_{p}\right]\left[\widehat{G}_{e}+I_{p}\right]^{-1}$ and $|\sigma(\circ)|$ denotes the absolute eigenspectrum of (o). Based on the dual concept of the digital redesign, the analog observer gain $L_{c}$ in (67a) 


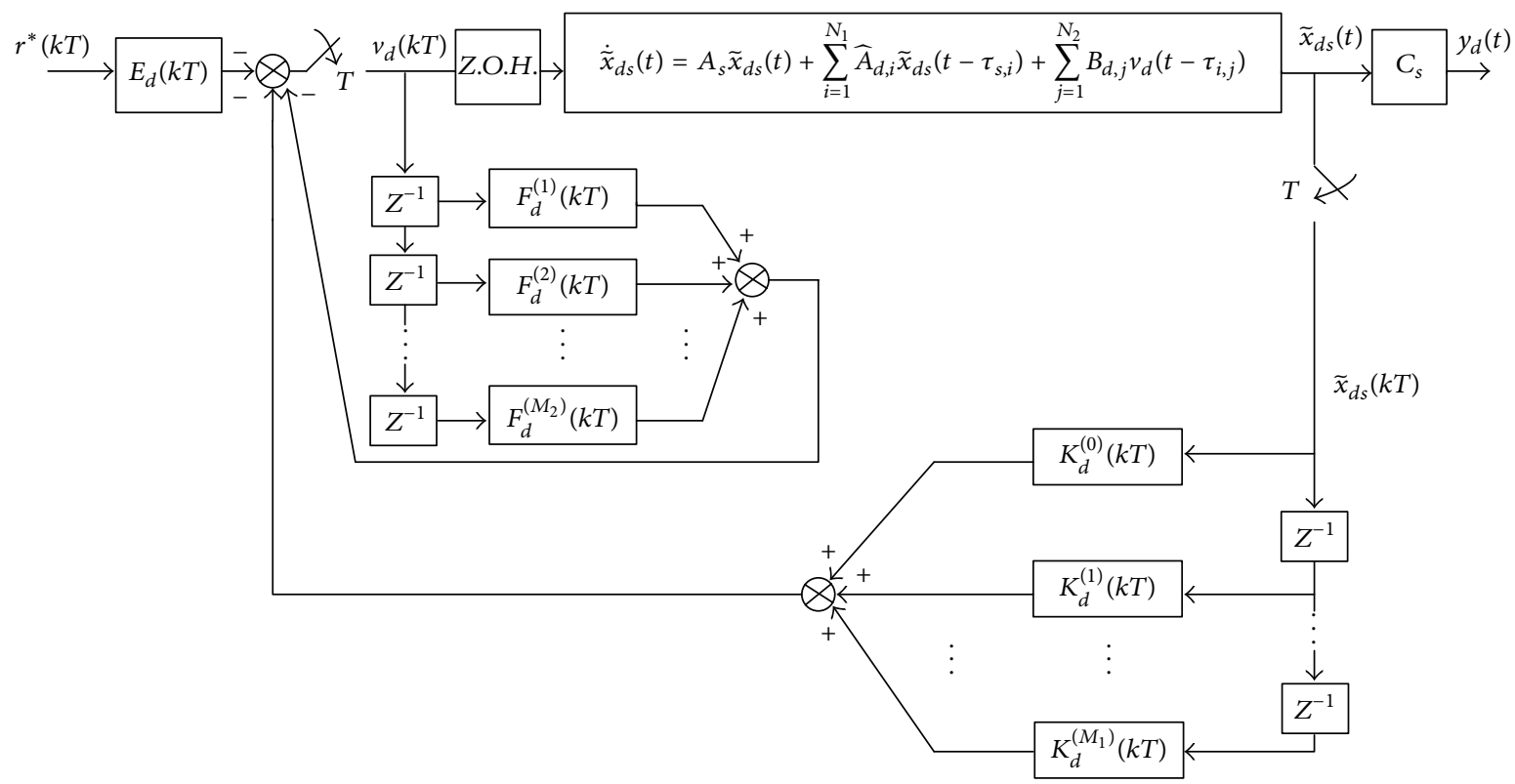

FIGURE 1: Digital redesign for time-delay singular system.

and the digitally redesigned observer gain $L_{d}$ in (66a) can be represented, respectively, as

$$
\begin{gathered}
L_{c}=P_{o b} \widehat{C}_{e}^{T} R^{-1}, \\
L_{d}=\left(\widehat{G}_{e}-I_{p}\right) \widehat{A}^{-1} L_{c}\left[I+\widehat{C}_{e}\left(\widehat{G}_{e}-I_{p}\right) \widehat{A}^{-1} L_{c}\right]^{-1},
\end{gathered}
$$

where $P_{o b}$ is the positive-definite and symmetric solution of the following Riccati equation:

$$
\widehat{A} P_{o b}+P_{o b} \widehat{A}^{T}-P_{o b} \widehat{C}_{e}^{T} R^{-1} \widehat{C}_{e} P_{o b}+\widehat{C}_{e}^{T} Q \widehat{C}_{e}=0,
$$

in which $Q \geq 0$ and $R>0$ with appropriate dimensions.

Owing to the extended virtual state vector in (40), the matrix $\widehat{G}_{e}$ in (41a) and (41b) and (67a), (67b), and (67c) is singular. The matrices $\widehat{A}$ and $\widehat{B}$ in (67a), (67b), and (67c) cannot be directly determined. To solve this problem, an alternative is derived via the matrix sign function method $[23,24]$ as follows.

Following the procedures shown in Section 3 [23, 24], the transformed matrix is

$$
\widehat{G}_{b}=\left(\widehat{G}_{e}-\zeta_{2} I_{p}\right)\left(\widehat{G}_{e}+\zeta_{2} I_{p}\right)^{-1},
$$

where $\widehat{G}_{e} \in \Re^{p \times p}$ and $\zeta_{2}$ is a radius of a circle from the origin of the coordinates. Additionally, the associated matrix sign functions are

$$
\begin{gathered}
\operatorname{Sign}\left(\widehat{G}_{b}\right)=\widehat{G}_{b}\left(\sqrt[2]{\widehat{G}_{b}^{2}}\right)^{-1}, \\
\operatorname{Sign}^{-}\left(\widehat{G}_{b}\right)=\frac{1}{2}\left(I_{p}-\operatorname{Sign}\left(\widehat{G}_{b}\right)\right), \\
\operatorname{Sign}^{+}\left(\widehat{G}_{b}\right)=\frac{1}{2}\left(I_{p}+\operatorname{Sign}\left(\widehat{G}_{b}\right)\right),
\end{gathered}
$$

respectively. A fast and stable algorithm for computing the matrix sign function $[23,24]$ is given as follows.

For the order of the desired convergence rate $r=2$, one has

$$
\begin{gathered}
Q(l+1)=\frac{1}{2}\left[Q(l)+Q^{-1}(l)\right], \\
Q(0)=\widehat{G}_{b}, \\
\lim _{l \rightarrow \infty} Q(l)=\operatorname{Sign}\left(\widehat{G}_{b}\right), \quad \text { for } l=0,1,2, \ldots
\end{gathered}
$$

By $[19,20]$, a transformation matrix $T_{m}$ can be found such that

$$
\widehat{G}_{m}=T_{m}^{-1} \widehat{G}_{e} T_{m}=\left[\begin{array}{c|c}
\widehat{G}_{m 1} & 0_{(p-g) \times g} \\
\hline 0_{g \times(p-g)} & \widehat{G}_{m 2}
\end{array}\right],
$$

where $\widehat{G}_{m 1}$ is a nonsingular matrix and $\widehat{G}_{m 2}$ is a singular matrix whose eigenvalues are all null. Finally, the matrix $\widehat{A}$ is obtained by the following equation:

$$
\widehat{A}=T_{m} \widehat{G}_{\ln } T_{m}^{-1}=T_{m}\left[\begin{array}{c|c}
\widehat{G}_{\ln 1} & 0 \\
\hline 0 & \widehat{G}_{\ln 2}
\end{array}\right] T_{m}^{-1},
$$

where $\widehat{G}_{\ln 1}=(1 / T) \ln \left(\sqrt[3]{\widehat{G}_{m 1}}\right)^{3}=(3 / T) \ln \left(\sqrt[3]{\widehat{G}_{m 1}}\right)$ and $\widehat{G}_{\ln 2}=v I_{g}$, in which $v$ is a large negative real constant. The algorithm for finding $\ln \left(\sqrt[3]{\widehat{G}_{m 1}}\right)$ in (76a) can be found in (67c) [19]. If the matrix $\widehat{G}_{m 1}$ has any negative real eigenvalue, then the principal third root of $\widehat{G}_{m 1}$ is not defined for $\arg \left(\lambda_{i}\right) \neq \pi$ $[23,24]$. The first part $\widehat{G}_{\ln 1}$ in $(76 a)$ can be rewritten as

$$
\widehat{G}_{\ln 1}=\frac{3}{T} \ln \left(\sqrt[3]{\widehat{G}_{m 1}}\right)=\frac{3}{T} \ln \left[\left(\sqrt[3]{\widehat{G}_{m 1} e^{i \theta}}\right) e^{-(i \theta / 3)}\right],
$$


where the matrix $\widehat{G}_{m 1}$ is rotated by a small positive real angle $\theta$. The second part $\widehat{G}_{\ln 2}$ in (76a) is utilized to recover the property $\ln (0)=-\infty$. Matrix $\widehat{B}$ can be evaluated as

$$
\begin{aligned}
\widehat{B} & =\widehat{A}\left(\widehat{G}_{e}-I_{p}\right)^{-1} \widehat{H}_{e} \\
& =\left[\left(\widehat{G}_{e}-I_{p}\right) \widehat{A}^{-1}\right]^{-1} \widehat{H}_{e} \\
& =\left(T I_{p}+\frac{\widehat{A} T^{2}}{2 !}+\frac{\widehat{A}^{2} T^{3}}{3 !}+\cdots\right)^{-1} \widehat{H}_{e} .
\end{aligned}
$$

Substituting (76a) and (76b) into the Riccati equation (70) and solving it yield the observer gain matrices in (68) and (69). Figure 2 presents the implementation of the observerbased suboptimal tracker for the time-delay singular system.

\section{An Illustrative Example}

Consider a continuous time-delay singular system, described in (1a) and (1b), with

$$
\begin{aligned}
& E=\left[\begin{array}{llllcc}
1 & 2 & 1 & 1 & -3 & -2 \\
0 & 2 & 2 & 1 & -3 & -3 \\
1 & 2 & 1 & 1 & -3 & -2 \\
1 & 2 & 1 & 3 & -5 & -4 \\
0 & 2 & 1 & 1 & -2 & -2 \\
1 & 0 & 0 & 0 & -1 & 0
\end{array}\right], \quad A=I_{6} \\
& \widehat{A}_{1}=\left[\begin{array}{cccccc}
0.447 & 0 & 0 & 0 & 0.447 & 0 \\
0 & 0.2236 & -0.1118 & 0 & -0.447 & 0 \\
0 & 0 & 0.2236 & 0 & 0 & 0 \\
0 & 0 & 0 & 0.447 & -0.8944 & 0 \\
0 & 0 & 0 & 0 & 0.447 & 0 \\
0 & 0 & 0 & 0 & -0.8944 & 0.447
\end{array}\right] \text {, } \\
& B_{1}=\left[\begin{array}{cccccc}
1 & 0 & 0 & 0 & 0 & -1 \\
0 & 0 & -1 & 1 & 0 & 0
\end{array}\right]^{T}, \quad C=\left[\begin{array}{llllll}
1 & 0 & 1 & 0 & 0 & 0 \\
0 & 1 & 0 & 1 & 0 & 0
\end{array}\right] \text {, } \\
& N_{1}=N_{2}=1, \quad \tau_{s 1}=0, \quad \tau_{i 1}=\tau_{o}=0.5 \times T \text {. }
\end{aligned}
$$

Let the sampling period $T=0.01(\mathrm{~s})$ and apply the reference input $r(t)=[0.5 \sin (t) \quad 0.5 \cos (t)]^{T}$ to the system. The initial condition is $x_{c}(0)=(M V \widetilde{M})\left[\begin{array}{ll}\tilde{x}_{s}^{T}(0) & \tilde{x}_{f}^{T}(0)\end{array}\right]^{T}=$ $\left[\begin{array}{llllll}0 & 0 & 0 & 0 & 0 & 0\end{array}\right]^{T}, \tilde{x}_{s}(0)=\left[\begin{array}{llll}0 & 0 & 0 & 0\end{array}\right]^{T}$, and $\tilde{x}_{f}=\left[\begin{array}{ll}0 & 0\end{array}\right]^{T}$.

Since $0 \times E+A=I_{6}$, and according to the definition of the standard form, $\{E, A\}$ is in standard form. If $\alpha=0$ and $\beta=1$ are set, then $E_{n}=E, A_{n}=A, \widehat{A}_{n, 1}=\widehat{A}_{1}$, and $B_{n, 1}=B_{1}$. Since $E_{n}$ is singular, $E_{n}$ includes some zero eigenvalues, and the bilinear transform must be performed to find the similarity transformation matrix $M$ of $E_{n}$. Assume $\omega=0.5$; the algorithm that was described in Section 3 yields,

$$
\widetilde{E}_{n}=\left[\begin{array}{cccccc}
0.3333 & 1.6 & -2.4 & 0.16 & 0.9067 & 2.24 \\
0 & 0.6 & 1.6 & 0.16 & -1.76 & -1.76 \\
1.3333 & 1.6 & -3.4 & 0.16 & 0.9067 & 2.24 \\
1.3333 & 1.6 & -2.4 & 0.76 & -0.6933 & 0.64 \\
0 & 1.6 & -2.4 & 0.16 & 1.24 & 2.24 \\
1.3333 & 0 & 0 & 0 & -1.3333 & -1
\end{array}\right],
$$

$$
\begin{aligned}
& \operatorname{sign}\left(\widetilde{E}_{n}\right)=\left[\begin{array}{cccccc}
1 & 2 & 2 & 0 & -4 & -2 \\
0 & 1 & 2 & 0 & -2 & -2 \\
2 & 2 & 1 & 0 & -4 & -2 \\
2 & 2 & 2 & 1 & -6 & -4 \\
0 & 2 & 2 & 0 & -3 & -2 \\
2 & 0 & 0 & 0 & -2 & -1
\end{array}\right], \\
& M=\left[\begin{array}{cccccc}
1 & 1 & 0 & 0 & -1 & -1 \\
0 & 1 & 0 & 0 & 0 & -1 \\
1 & 1 & 0 & -1 & -1 & 0 \\
1 & 1 & 1 & -1 & -1 & -1 \\
0 & 1 & 0 & 0 & -1 & -1 \\
1 & 0 & 0 & -1 & 0 & 0
\end{array}\right]
\end{aligned}
$$

Based on Section 3.1 and Appendices, the time-delay singular system can be decomposed as follows:

$$
\begin{gathered}
\dot{\tilde{x}}_{s}(t)=A_{s} \tilde{x}_{s}(t)+\widehat{A}_{d, 1} \tilde{x}_{s}\left(t-\tau_{s, 1}\right)+B_{d, 1} v_{c}\left(t-\tau_{i, 1}\right), \\
y_{c}(t)=C_{1} \tilde{x}_{s}\left(t-\tau_{o}\right)-D_{1} v_{c}\left(t-\tau_{i, 1}\right),
\end{gathered}
$$

where

$$
\begin{aligned}
& A_{s}=\left[\begin{array}{cccc}
1 & 0 & 0 & -0.5 \\
0 & 0.5 & -0.25 & -0.5 \\
0 & 0 & 0.5 & 0 \\
0 & 0 & 0 & 0.5
\end{array}\right] \\
& \widehat{A}_{d, 1}=\left[\begin{array}{cccc}
0.4472 & 0 & 0 & -0.2236 \\
0 & 0.2236 & -0.1118 & -0.2236 \\
0 & 0 & 0.2236 & 0 \\
0 & 0 & 0 & 0.2236
\end{array}\right] \text {, } \\
& B_{d, 1}=\left[\begin{array}{cc}
0.5 & 0.5 \\
-0.25 & -0.25 \\
0.5 & 0.5 \\
0.5 & -0.5
\end{array}\right] \\
& C_{1}=\left[\begin{array}{llll}
1 & 0 & 1 & 0 \\
1 & 0 & 0 & 0
\end{array}\right] \text {, } \\
& D_{1}=\left[\begin{array}{cc}
0 & 2 \\
0.5 & 1.5
\end{array}\right] \text {, }
\end{aligned}
$$

and the other parameters are listed below:

$$
\begin{gathered}
\widetilde{M}=\left[\begin{array}{llllll}
1 & 0 & 0 & 0 & 0 & 0 \\
0 & 1 & 0 & 0 & 0 & 0 \\
0 & 0 & 1 & 0 & 0 & 0 \\
0 & 0 & 0 & 1 & 1 & 0 \\
0 & 0 & 0 & 0.5 & 0 & 1 \\
0 & 0 & 0 & 1 & 0 & 0
\end{array}\right], \\
\Lambda_{1,1}=\left[\begin{array}{ccccc}
0.4472 & 0 & \eta=-1, \\
0 & 0.1491 & -0.0994 & -0.1988 \\
0 & 0 & 0.1491 & 0 \\
0 & 0 & 0 & 0.1491
\end{array}\right],
\end{gathered}
$$




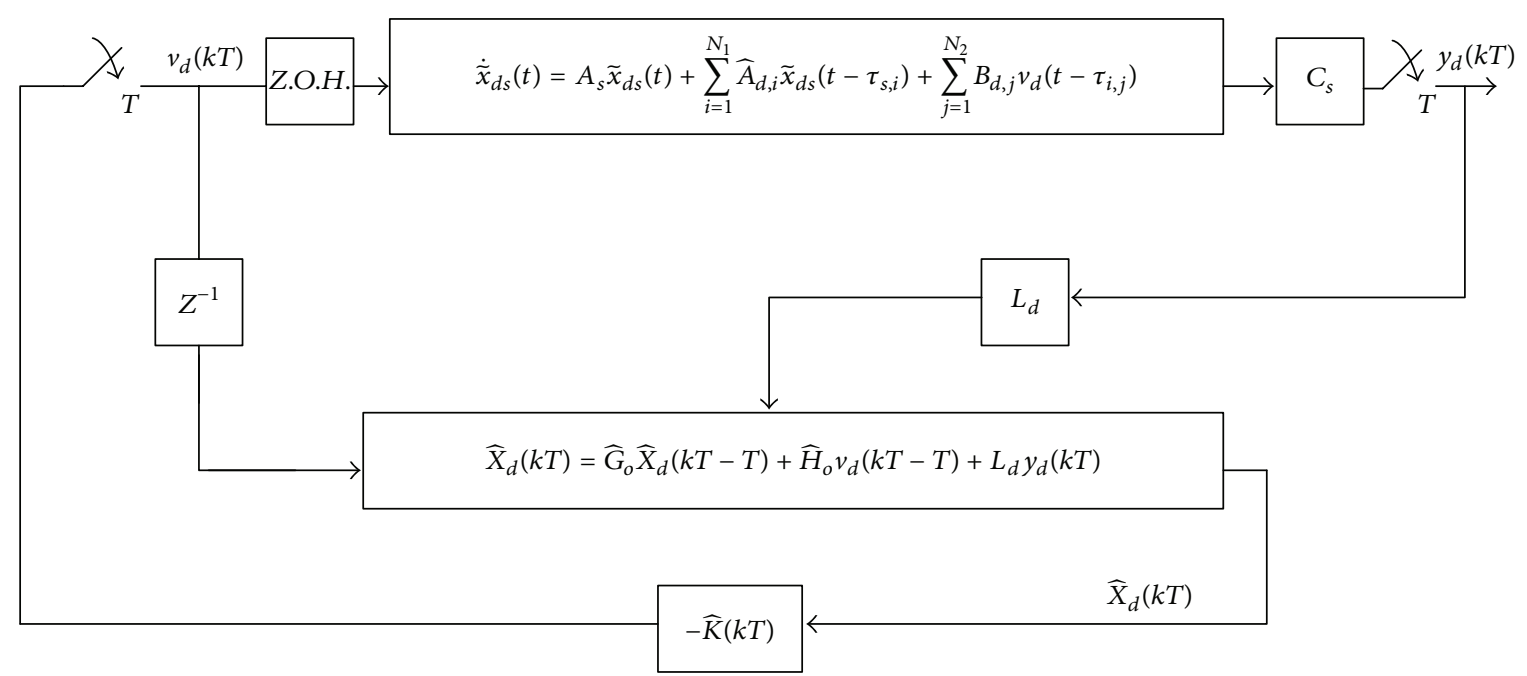

FIGURE 2: Observer-based suboptimal tracker for the digitally redesigned time-delay singular system.
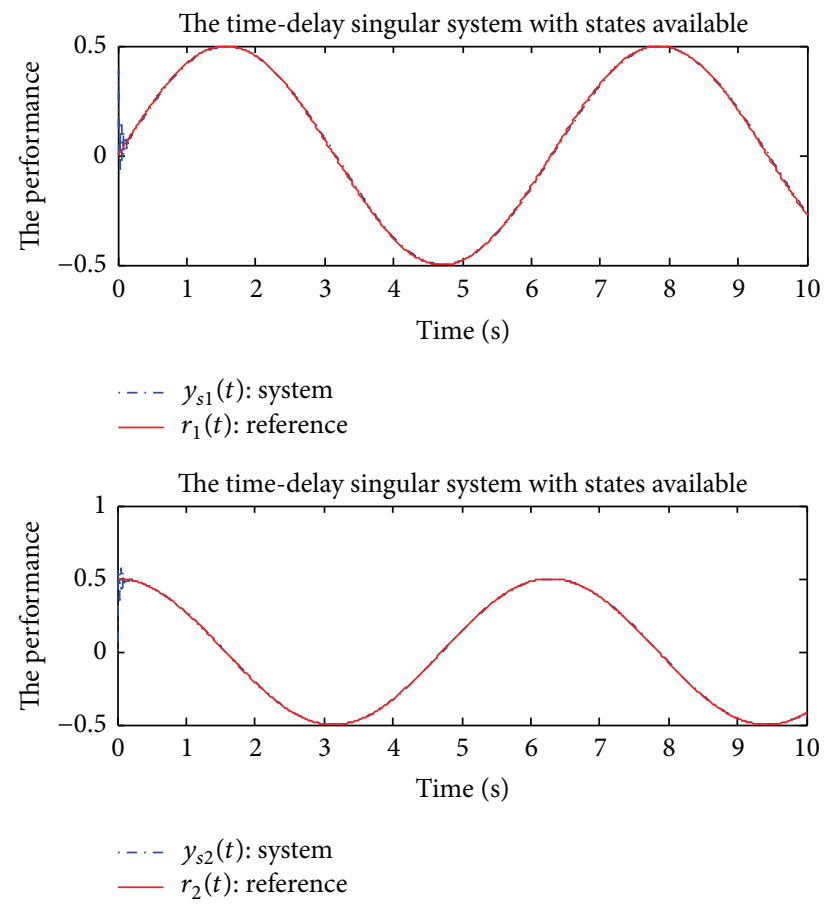

FIgURE 3: Output responses of time-delay singular system with states available by the new digital redesign approach.

$$
\begin{gathered}
\Lambda_{2,1}=\left[\begin{array}{cc}
-0.4472 & 0 \\
0 & -0.4472
\end{array}\right], \\
B_{s k, 1}=\left[\begin{array}{cc}
0.3333 & 0.6667 \\
-0.3333 & -0.1111 \\
0.3333 & 0.3333 \\
0.3333 & -0.3333
\end{array}\right], \\
V=I_{6}, \quad K_{f, 1}=\left[\begin{array}{ccc}
0 & 1 & 0 \\
0 & -1 & 0
\end{array}\right] .
\end{gathered}
$$

Following the proposed method in Section 3, the schemes of Figures 1 and 2 are implemented. For simplification, the numerical analysis is not presented and Figures 3 and 4 show the results of the simulation.

Comparing with the offline observer/Kalman filter identification (OKID) method, the advantages of the proposed approach can be shown in [33,34]. Following [33, 34], let the unknown system (80a) and (80b) be excited by the whitenoise control force $u(t)=\left[\begin{array}{ll}u_{1}(t) & u_{2}(t)\end{array}\right]^{T}$ with a zero mean and covariance $\operatorname{diag}\left[\operatorname{cov}\left(u_{1}(t)\right), \operatorname{cov}\left(u_{2}(t)\right)\right]=\operatorname{diag}\left[\begin{array}{ll}0.2 & 0.2\end{array}\right]$. The input-output sampled data is given in Figure 5. 


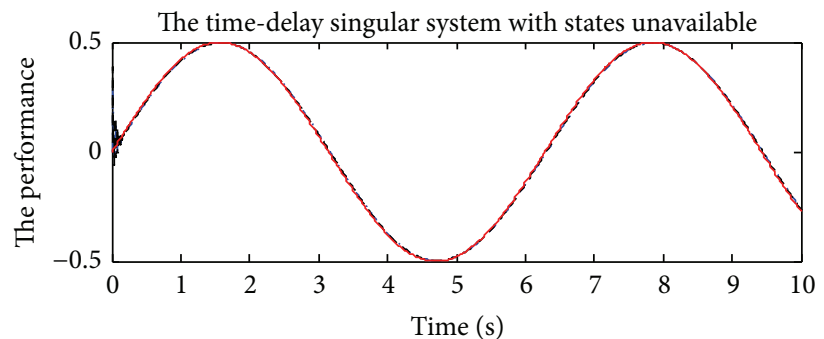

-..- $y_{o 1}(t)$ : observer

$--y_{s 1}:$ system

$r_{1}(t)$ : reference

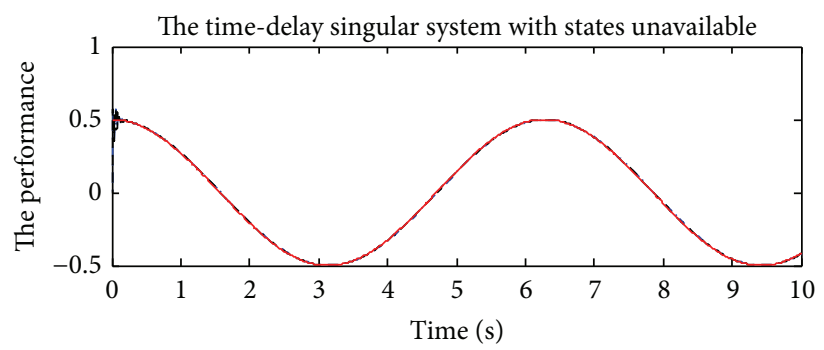

-.. $y_{o 2}(t)$ : observer

- - $y_{s 2}:$ system

$r_{2}(t)$ : reference

FIGURE 4: Output responses of time-delay singular system with states unavailable by new observer-based suboptimal approach.

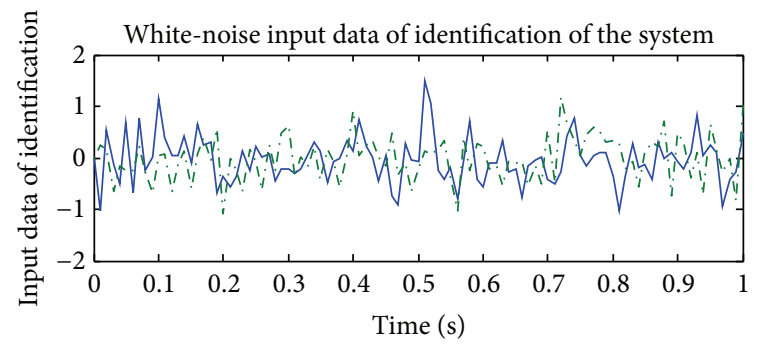

$u_{s 1}(k T)$

$\cdot-\cdot u_{s 2}(k T)$

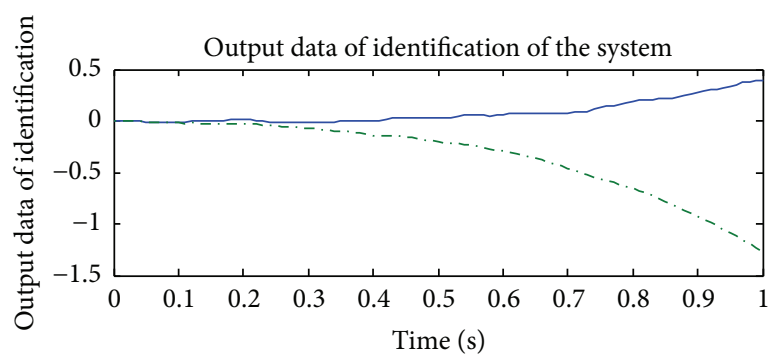

$-y_{s 1}(k T)$

-.. $y_{s 2}(k T)$

Figure 5: System $I / O$ data for identification.

The identified system $(\widehat{G}, \widehat{H}, \widehat{C})$ and observer gain $(F)$ matrices for the unknown system (80a) and (80b) are given as

$$
\widehat{G}=\left[\begin{array}{ccccc}
8.7538 & -4.3746 & 8.9478 & -0.5128 & 0 \\
10.2956 & 5.5361 & 1.4682 & -3.6730 & 0 \\
-19.5769 & 9.2613 & -19.7421 & 9.5377 & 0 \\
-0.0821 & 0.6656 & -1.0711 & -359.3919 & 0 \\
0 & 0 & 0 & 0 & -112.9831
\end{array}\right],
$$

$$
\begin{gathered}
\widehat{H}=\left[\begin{array}{cc}
-0.2470 & -0.2116 \\
-0.8808 & -0.8879 \\
-0.5713 & -0.5007 \\
22.2478 & 21.6048 \\
0 & 0
\end{array}\right], \\
\widehat{C}=\left[\begin{array}{ccccc}
-1.0867 & -0.5871 & 0.7911 & -0.0579 & 0 \\
0.5676 & -0.5710 & -0.4333 & -0.0488 & 0
\end{array}\right],
\end{gathered}
$$



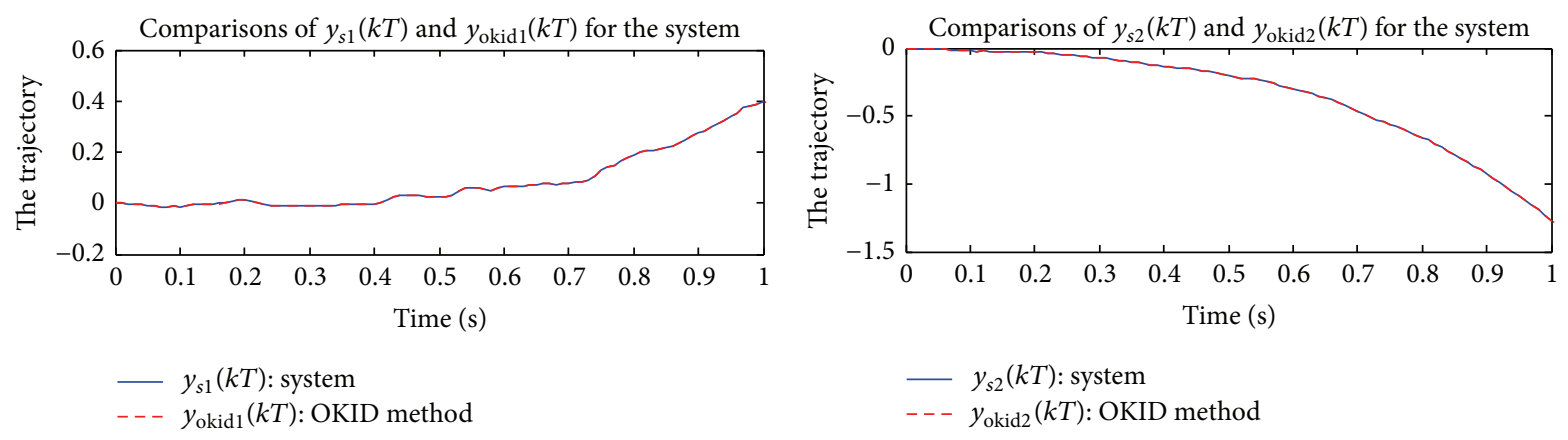

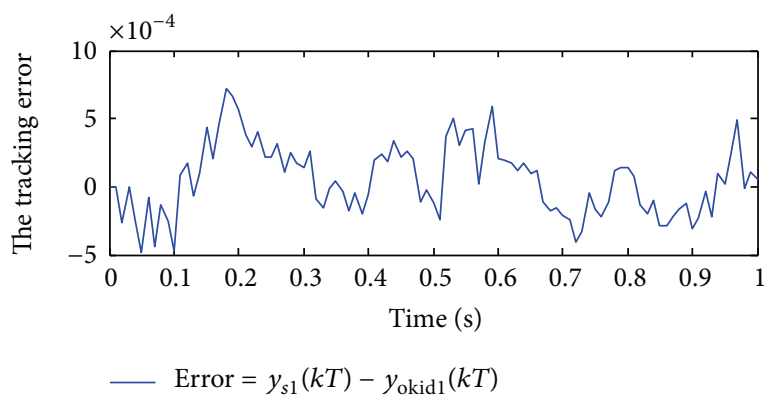

(a)

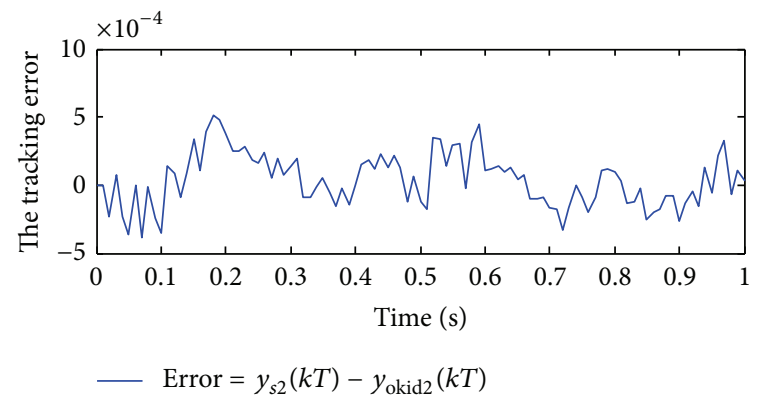

(b)

FIGURE 6: (a) The comparison between the system output $y_{s 1}(k T)$ and its observer-based output $y_{\text {okid } 1}(k T)$ by OKID. (b) The comparison between the system output $y_{s 2}(k T)$ and its observer-based output $y_{\text {okid2 }}(k T)$ by OKID.
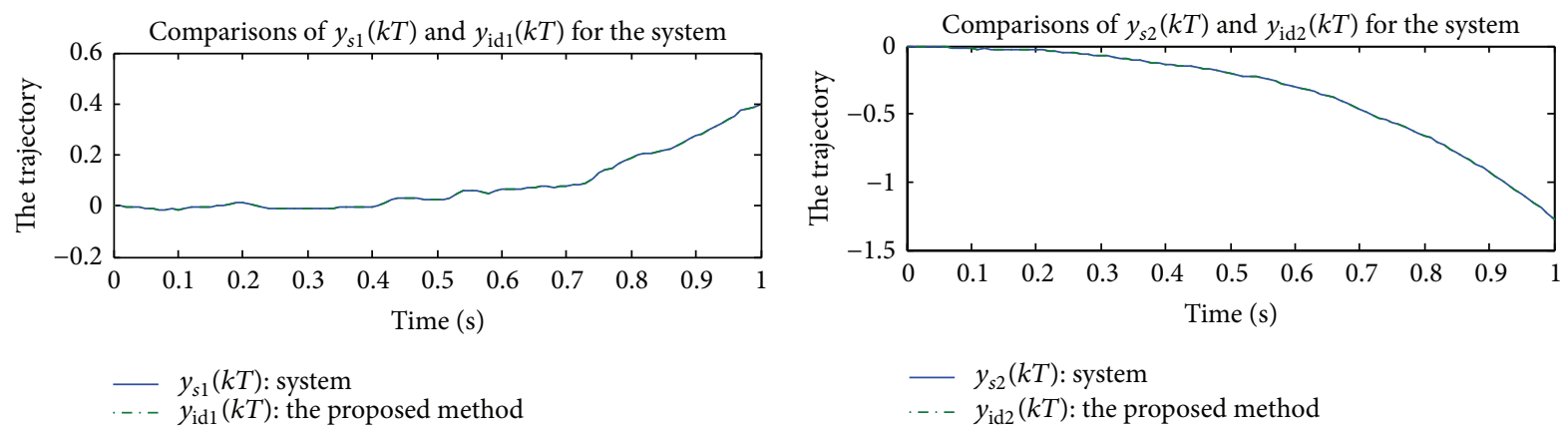

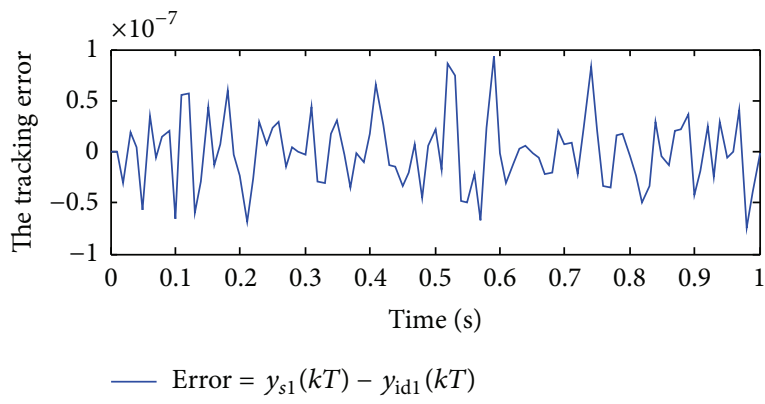

(a)

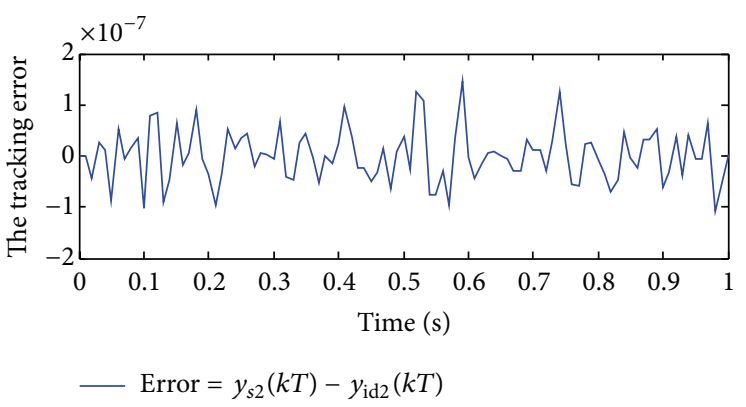

(b)

Figure 7: (a) The comparison between the system output $y_{s 1}(k T)$ and its observer-based output $y_{\text {id1 }}(k T)$ by the proposed method. (b) The comparison between the system output $y_{s 2}(k T)$ and its observer-based output $y_{\mathrm{id} 2}(k T)$ by the proposed method. 


$$
F=\left[\begin{array}{cc}
1.4216 & -2.0615 \\
0.7740 & 1.0579 \\
0.5564 & -0.8225 \\
-0.0043 & -0.0041 \\
0 & 0
\end{array}\right]
$$

Then, the observer-based outputs by OKID compared with the actual system outputs for the unknown system (80a) and $(80 \mathrm{~b})$ are shown in Figure 6.

To overcome the effect of modeling error, an improved observer (69) with the high-gain property has been proposed in this paper, where the observer gain matrices are given as

$$
\begin{aligned}
& L_{d}=\left[\begin{array}{cccccc}
-0.3231 & -0.5708 & 0.3103 & -0.1051 & 0 & -0.0628 \\
0.4008 & -1.0446 & -0.4013 & -0.1646 & 0 & 0.1289
\end{array}\right. \\
& \begin{array}{llllll}
-0.0833 & -0.1119 & 6.4407 & 0 & 1.1006 & 0.1373
\end{array} \\
& \begin{array}{llllll}
-0.1585 & -0.4047 & 10.2422 & 0 & 1.6436 & 0.3760
\end{array} \\
& \left.\begin{array}{lllllll}
-16.6049 & 228.3244 & 0.0003 & 0.0124 & 0.0119 & 0 & 0
\end{array}\right]^{T} \text {. } \\
& \left.\begin{array}{lllllll}
-25.8086 & 363.6177 & 0.0001 & 0.0191 & 0.0184 & 0 & 0
\end{array}\right] \text {. }
\end{aligned}
$$

Then, the comparisons between the actual outputs and the proposed method outputs for the unknown system (80a) and (80b) are shown in Figure 7.

Obviously, the proposed method is better than OKID method on the tracking error performance from Figures 6 and 7.

\section{Conclusion}

This paper presents a systematic methodology for developing novel observer-based suboptimal digital trackers for a class of time-delay singular systems. The time-delay property and singular system have been attracting more attention in recent years. The proposed controller and observer depend on the concepts of optimal control and the digital redesign with high-gain property to ensure effective tracking and favorable state matching performance. In future works, we will pay more attention to the online application and the realtime implementation of fault tolerant control system with performance optimization by using the proposed methods.

\section{Appendices}

\section{A. Transformation of the Time-Delay Singular Systems}

The following steps yield the preliminary feedback gain $K_{f, j}$ and prove that $K_{f, j}$ can eliminate impulsive modes.

Let

$$
\bar{x}_{c}(t)=V \hat{x}_{c}(t)
$$

where $\widehat{x}_{c}(t)=\left[\widehat{x}_{c, s}^{T}(t), \widehat{x}_{c, f}^{T}(t)\right]^{T}=\left[\bar{x}_{c, s}^{T}(t),\left(U^{-1} \bar{x}_{c, f}(t)\right)^{T}\right]^{T}$ and $V=\left[\begin{array}{l|l}I_{k} & O \\ \hline O & U\end{array}\right] . U$ is a modal matrix of $\bar{E}_{f}$ with dimension $(n-k) \times(n-k)$ such that $U^{-1} \bar{E}_{f} U$ is in the Jordan block form. Substituting (A.1) into (9) and multiplying by $V^{-1}$ yield the following equation:

$$
\begin{aligned}
& {\left[\begin{array}{c|c}
I_{k} & O \\
\hline O & \widehat{E}_{f}
\end{array}\right] \dot{\hat{x}}_{c}(t)} \\
& =\left[\begin{array}{c|c}
\widehat{A}_{s} & O \\
\hline O & I_{n-k}
\end{array}\right] \widehat{x}_{c}(t) \\
& \quad+\sum_{i=1}^{N_{1}}\left[\begin{array}{c|c}
\widehat{A}_{1, i} & O \\
\hline O & \widehat{A}_{2, i}
\end{array}\right] \widehat{x}_{c}\left(t-\tau_{s, i}\right) \\
& \quad+\sum_{j=1}^{N_{2}}\left[\frac{\widehat{B}_{s, j}}{\widehat{B}_{f, j}}\right] u_{c}\left(t-\tau_{i, j}\right),
\end{aligned}
$$

where $\widehat{E}_{f}=U^{-1} \bar{E}_{f} U, \widehat{A}_{s}=\bar{A}_{s}, \widehat{A}_{1, i}=\widetilde{A}_{1, i}, \widehat{A}_{2, i}=U^{-1} \widetilde{A}_{2, i} U$, $\widehat{B}_{s, j}=\bar{B}_{s, j}$, and $\widehat{B}_{f, j}=U^{-1} \bar{B}_{f, j}$. Notably, $\widehat{E}_{f}$ is in the Jordan block form with $d$ blocks of sizes $\mu_{1}, \mu_{2}, \ldots, \mu_{d}$, where $\sum_{i=1}^{d} \mu_{i}=$ column (row) number of $\widehat{E}_{f}$. In (A.2), the statedelay $\hat{x}_{c}\left(t-\tau_{s, i}\right)$ can be equal to $W_{s, i} \hat{x}_{c}(t)$, where $W_{s, i}$ is a block diagonal $\left\{\psi_{1, i}, \psi_{2, i}\right\}$. Therefore, (A.2) can be rewritten as

$$
\begin{aligned}
& {\left[\begin{array}{c|c}
I_{k} & O \\
\hline \mathrm{O} & \widehat{E}_{f}
\end{array}\right] \dot{\hat{x}}_{c}(t)} \\
& =\left[\begin{array}{c|c}
\widehat{A}_{s}+\sum_{i=1}^{N_{1}} \widehat{A}_{1, i} \psi_{1, i} & O \\
\hline O & I+\sum_{i=1}^{N_{1}} \widehat{A}_{2, i} \psi_{2, i}
\end{array}\right] \widehat{x}_{c}(t) \\
& +\sum_{j=1}^{N_{2}}\left[\frac{\widehat{B}_{s, j}}{\widehat{B}_{f, j}}\right] u_{c}\left(t-\tau_{i, j}\right) \\
& \triangleq\left[\begin{array}{c|c}
A_{s 1} & O \\
\hline O & A_{s 2}
\end{array}\right] \widehat{x}_{c}(t)+\sum_{j=1}^{N_{2}}\left[\frac{\widehat{B}_{s, j}}{\widehat{B}_{f, j}}\right] u_{c}\left(t-\tau_{i, j}\right) .
\end{aligned}
$$

From (A.3), the fast subsystem is

$$
\widehat{E}_{f} \dot{\hat{x}}_{c, f}(t)=A_{s 2} \widehat{x}_{c, f}(t)+\sum_{j=1}^{N_{2}} \widehat{B}_{f, j} u_{c}\left(t-\tau_{i, j}\right),
$$

so

$$
\widehat{E}_{f}^{*} \dot{\hat{x}}_{c, f}(t)=\widehat{x}_{c, f}(t)+\sum_{j=1}^{N_{2}} \widehat{B}_{f, j}^{*} U_{c, j}(t),
$$

where $\widehat{E}_{f}^{*}=A_{s 2}^{-1} \widehat{E}_{f}, \widehat{B}_{f, j}^{*}=A_{s 2}^{-1} \widehat{B}_{f, j}$, and $U_{c, j}(t)=u_{c}\left(t-\tau_{i, j}\right)$.

Taking the Laplace transformation of the fast subsystem (A.5), one obtains

$$
\begin{aligned}
\widehat{X}_{c, f}(s) & =\left(s \widehat{E}_{f}^{*}-I_{n-k}\right)^{-1}\left(\widehat{E}_{f}^{*} \widehat{x}_{c, f}(0)+\sum_{j=1}^{N_{2}} \widehat{B}_{f, j}^{*} U_{c, j}(s)\right) \\
& =-\sum_{i=0}^{l-1} s^{i}\left(\widehat{E}_{f}^{*}\right)^{i}\left(\widehat{E}_{f}^{*} \widehat{x}_{c, f}(0)+\sum_{j=1}^{N_{2}} \widehat{B}_{f, j}^{*} U_{c, j}(s)\right) .
\end{aligned}
$$


Taking the inverse Laplace transformation of the above equation, one has

$$
\begin{aligned}
\widehat{x}_{c, f}(t)= & -\sum_{i=1}^{l-1}\left(\widehat{E}_{f}^{*}\right)^{i} \widehat{x}_{c, f}(0) \delta^{(i-1)}(t) \\
& -\sum_{i=0}^{l-1} \sum_{j=1}^{N_{2}}\left(\widehat{E}_{f}^{*}\right)^{i} \widehat{B}_{f, j}^{*} U_{c, j}^{(i)}(t),
\end{aligned}
$$

where $\delta(t)$ and $\delta^{(i)}(t)$ denote the delta function and the $i$ th derivative of the delta function, respectively. From the above equation, the impulsive modes of the fast state are induced from inconsistent initial conditions of the fast state or discontinuous control input (or its derivatives). By [12], determination of the preliminary feedback gain $K_{f, j}=$ $\left[k_{1, j}, k_{2, j}, \ldots, k_{n-k, j}\right]_{m \times(n-k)}$, where $k_{\xi, j}$ is of dimension $m \times 1$ for $\xi=1,2, \ldots,(n-k)$, is summarized as follows.

(1) If $\mu_{i} \geq 1$, where $1 \leq i \leq d$, and its corresponding Jordan block is a null matrix, then

$$
\begin{gathered}
k_{\mu_{1}+\mu_{2}+\cdots+\mu_{i-1}+1, j}=O_{m \times 1}, \\
k_{\mu_{1}+\mu_{2}+\cdots+\mu_{i-1}+2, j}=O_{m \times 1}, \\
\vdots \\
k_{\mu_{1}+\mu_{2}+\cdots+\mu_{i-1}+\mu_{i}, j}=O_{m \times 1}
\end{gathered}
$$

(2) If $\mu_{i}>1$, where $1 \leq i \leq d$, and its corresponding Jordan block is not a null matrix, then

$$
\begin{gathered}
k_{\mu_{1}+\mu_{2}+\cdots+\mu_{i-1}+1, j}=\left[\begin{array}{c}
\delta\left(\widehat{b}_{\left(\mu_{1}+\mu_{2}+\cdots+\mu_{i}\right) 1, j}\right) \\
\delta\left(\widehat{b}_{\left(\mu_{1}+\mu_{2}+\cdots+\mu_{i}\right) 2, j}\right) \\
\vdots \\
\delta\left(\widehat{b}_{\left(\mu_{1}+\mu_{2}+\cdots+\mu_{i}\right) m, j}\right)
\end{array}\right], \\
k_{\mu_{1}+\mu_{2}+\cdots+\mu_{i-1}+2, j}=O_{m \times 1}, \\
\vdots \\
k_{\mu_{1}+\mu_{2}+\cdots+\mu_{i-1}+\mu_{i}, j}=O_{m \times 1},
\end{gathered}
$$

where

$$
\begin{gathered}
\widehat{B}_{f, j}^{*} \triangleq\left[\begin{array}{c}
\widehat{b}_{(k+1), j} \\
\widehat{b}_{(k+2), j} \\
\vdots \\
\widehat{b}_{n, j}
\end{array}\right]_{(n-k) \times m}, \\
\widehat{b}_{i, j} \triangleq\left[\widehat{b}_{i 1, j}, \widehat{b}_{i 2, j}, \ldots, \widehat{b}_{i m, j}\right]_{1 \times m}, \\
\delta\left(\widehat{b}_{i \xi, j}\right) \triangleq \begin{cases}0, & \text { if } \widehat{b}_{i \xi, j}=0, \\
1, & \text { if } \widehat{b}_{i \xi, j}>0, \quad \xi=1,2, \ldots, m . \\
-1, & \text { if } \widehat{b}_{i \xi, j}<0,\end{cases}
\end{gathered}
$$

Let

$$
\begin{aligned}
U_{c, j}(t)=u_{c}\left(t-\tau_{i, j}\right) & =-K_{f, j} \widehat{x}_{c, f}(t)+V_{c, j}(t) \\
& =-K_{f, j} \widehat{x}_{c, f}(t)+v\left(t-\tau_{i, j}\right) \\
& =-\left[O_{m \times k}, K_{f, j}\right] \widehat{x}_{c}(t)+v_{c}\left(t-\tau_{i, j}\right) .
\end{aligned}
$$

Substituting (A.11) into (A.2) yields (13).

\section{B. Output Transformation of the Time-Delay Singular Systems}

Equation (20b) can be decomposed as follows:

$$
\begin{aligned}
& 0=\tilde{x}_{f, s}(t)+\sum_{i=1}^{N_{1}} \Delta_{1, i} \tilde{x}_{f, s}\left(t-\tau_{s, i}\right)+\sum_{j=1}^{N_{2}} \bar{B}_{s, j}^{*} v_{c}\left(t-\tau_{i, j}\right), \\
& 0=\tilde{x}_{f, f}(t)+\sum_{i=1}^{N_{1}} \Delta_{2, i} \tilde{x}_{f, f}\left(t-\tau_{s, i}\right)+\sum_{j=1}^{N_{2}} \bar{B}_{f, j}^{*} v_{c}\left(t-\tau_{i, j}\right),
\end{aligned}
$$

where $\tilde{x}_{f}(t)=\left[\begin{array}{l}\tilde{x}_{f, s}(t) \\ \tilde{x}_{f, f}(t)\end{array}\right], \bar{B}_{f k, j}=\left[\begin{array}{c}\bar{B}_{s, j}^{*} \\ \bar{B}_{f, j}^{*}\end{array}\right]$, and $\eta \Lambda_{2, i}=$ block diagonal $\left\{\Delta_{1, i}, \Delta_{2, i}\right\}$ is assumed. Based on (B.la) and (B.1b), the following equations hold:

$$
\begin{gathered}
\sum_{i=1}^{N_{1}+1} \Delta_{1, i} \tilde{x}_{f, s}\left(t-\tau_{s, i}\right)=-\sum_{j=1}^{N_{2}} \bar{B}_{s, j}^{*} v_{c}\left(t-\tau_{i, j}\right), \\
\sum_{i=1}^{N_{1}+1} \Delta_{2, i} \tilde{x}_{f, f}\left(t-\tau_{s, i}\right)=-\sum_{j=1}^{N_{2}} \bar{B}_{f, j}^{*} v_{c}\left(t-\tau_{i, j}\right),
\end{gathered}
$$

where $i=k$ and $\Delta_{1, k}=\Delta_{1, k}=I, \tau_{s, k}=0$. Similarly, from (21a),

$$
\begin{aligned}
y_{c}(t) & =C_{1} \tilde{x}_{s}\left(t-\tau_{o}\right)+C_{2} \tilde{x}_{f}\left(t-\tau_{o}\right) \\
& =C_{1} \tilde{x}_{s}\left(t-\tau_{o}\right)+\left[\begin{array}{ll}
\widetilde{C}_{1} & \widetilde{C}_{2}
\end{array}\right]\left[\begin{array}{c}
\tilde{x}_{f, s}\left(t-\tau_{o}\right) \\
\tilde{x}_{f, f}\left(t-\tau_{o}\right)
\end{array}\right] \\
& =C_{1} \tilde{x}_{s}\left(t-\tau_{o}\right)+\widetilde{C}_{1} \tilde{x}_{f, s}\left(t-\tau_{o}\right)+\widetilde{C}_{2} \tilde{x}_{f, f}\left(t-\tau_{o}\right),
\end{aligned}
$$

where $C_{2}=\left[\begin{array}{ll}\widetilde{C}_{1} & \widetilde{C}_{2}\end{array}\right]$. From (B.3) denotes the following equation is satisfied:

$$
\begin{aligned}
\sum_{i=1}^{N_{1}+1} y_{c}\left(t-\tau_{i}^{*}\right)= & \sum_{i=1}^{N_{1}+1} C_{1} \widetilde{x}_{s}\left(t-\tau_{o}-\tau_{i}^{*}\right) \\
& +\sum_{i=1}^{N_{1}+1} \widetilde{C}_{1} \widetilde{x}_{f, s}\left(t-\tau_{o}-\tau_{i}^{*}\right) \\
& +\sum_{i=1}^{N_{1}+1} \widetilde{C}_{2} \widetilde{x}_{f, f}\left(t-\tau_{o}-\tau_{i}^{*}\right)
\end{aligned}
$$




$$
\begin{aligned}
= & \sum_{i=1}^{N_{1}+1} C_{1} \widetilde{x}_{s}\left(t-\tau_{s, i}\right) \\
& -\widetilde{C}_{1} \sum_{i=1}^{N_{1}+1} \sum_{j=1}^{N_{2}} \Delta_{1, i}^{-1} \bar{B}_{s, j}^{*} v_{c}\left(t-\tau_{i, j}\right) \\
& -\widetilde{C}_{2} \sum_{i=1}^{N_{1}+1} \sum_{j=1}^{N_{2}} \Delta_{2, i}^{-1} \bar{B}_{f, j}^{*} v_{c}\left(t-\tau_{i, j}\right) \\
= & \sum_{i=1}^{N_{1}+1} C_{1} \widetilde{x}_{s}\left(t-\tau_{s, i}\right)-\left[\widetilde{C}_{1} \widetilde{C}_{2}\right] \\
& \times \sum_{i=1}^{N_{1}+1} \sum_{j=1}^{N_{2}}\left[\begin{array}{cc}
\Delta_{1, i}^{-1} & O \\
O & \Delta_{2, i}^{-1}
\end{array}\right]\left[\begin{array}{c}
\bar{B}_{s, j}^{*} \\
\bar{B}_{f, j}^{*}
\end{array}\right] v_{c}\left(t-\tau_{i, j}\right) \\
= & \sum_{i=1}^{N_{1}+1} C_{1} \tilde{x}_{s}\left(t-\tau_{s, i}\right) \\
& -C_{2} \sum_{i=1}^{N_{1}+1} \sum_{j=1}^{N_{2}}\left(\eta \Lambda_{2, i}\right)^{-1} \bar{B}_{f k, j} v_{c}\left(t-\tau_{i, j}\right),
\end{aligned}
$$

where $\tau_{i}^{*}=\tau_{s, i}-\tau_{o}$. One of the terms $\tau_{i}^{*}$ in (B.4) is set to $\tau_{i}^{*}=0$ and (B.4) can be represented as

$$
\begin{aligned}
y_{c}(t) & +\sum_{i=1}^{N_{1}} y_{c}\left(t-\tau_{i}^{*}\right) \\
= & C_{1} \tilde{x}_{s}\left(t-\tau_{o}\right)-C_{2}\left(\eta \Lambda_{2, k}\right)^{-1} \\
& \times \sum_{j=1}^{N_{2}} \bar{B}_{f k, j} v_{c}\left(t-\tau_{i, j}\right)+\sum_{i=1}^{N_{1}} C_{1} \tilde{x}_{s}\left(t-\tau_{s, i}\right) \\
& -C_{2} \sum_{i=1}^{N_{1}} \sum_{j=1}^{N_{2}}\left(\eta \Lambda_{2, i}\right)^{-1} \bar{B}_{f k, j} v_{c}\left(t-\tau_{i, j}\right) \\
= & C_{1} \tilde{x}_{s}\left(t-\tau_{o}\right)-C_{2}(I)^{-1} \sum_{j=1}^{N_{2}} \bar{B}_{f k, j} v_{c}\left(t-\tau_{i, j}\right) \\
& +\sum_{i=1}^{N_{1}} C_{1} \tilde{x}_{s}\left(t-\tau_{s, i}\right) \\
& -C_{2} \sum_{i=1}^{N_{1}} \sum_{j=1}^{N_{2}}\left(\eta \Lambda_{2, i}\right)^{-1} \bar{B}_{f k, j} v_{c}\left(t-\tau_{i, j}\right) .
\end{aligned}
$$

From (B.5),

$$
y_{c}(t)=C_{1} \tilde{x}_{s}\left(t-\tau_{o}\right)-C_{2} \sum_{j=1}^{N_{2}} \bar{B}_{f k, j} v_{c}\left(t-\tau_{i, j}\right) .
$$

\section{Acknowledgment}

The authors would like to thank the National Science Council of the Republic of China, Taiwan, for financially supporting this research under Contract no. NSC 101-2511-S-197-002.

\section{References}

[1] J. Lin and Z. Gao, "Exponential admissibility and $H_{\infty}$ control of switched singular time-delay systems: an average dwell time approach," Journal of Applied Mathematics, vol. 2012, Article ID 482792, 28 pages, 2012.

[2] P. Wang, C. Han, and B. Ding, "Stability of discrete-time networked control systems and its extension for robust $H_{\infty}$ control," International Journal of Systems Science, vol. 44, no. 2, pp. 275-288, 2013.

[3] L. Xie, T. Liu, G. Lu, J. Liu, and S. T. C. Wong, "Stochastic robust stability analysis for Markovian jump discrete-time delayed neural networks with multiplicative nonlinear perturbations," in Advances in Neural Networks, vol. 3971 of Lecture Notes in Computer Science, pp. 172-178, Springer, 2006.

[4] X. Zhang, S. Li, and H. Li, "Structure and BIBO stability of a three-dimensional fuzzy two-term control system," Mathematics and Computers in Simulation, vol. 80, no. 10, pp. 1985-2004, 2010.

[5] J. S.-H. Tsai, C.-T. Wang, and L. S. Shieh, "Model conversion and digital redesign of singular systems," Journal of the Franklin Institute, vol. 330, no. 6, pp. 1063-1086, 1993.

[6] B. G. Mertzios, M. A. Christodoulou, B. L. Syrmos, and F. L. Lewis, "Direct controllability and observability time domain conditions of singular systems," IEEE Transactions on Automatic Control, vol. 33, no. 8, pp. 788-791, 1988.

[7] C.-J. Wang and H.-E. Liao, "Impulse observability and impulse controllability of linear time-varying singular systems," Automatica, vol. 37, no. 11, pp. 1867-1872, 2001.

[8] J. Yu, G. Sun, and H. R. Karimi, "Fault-reconstruction-based cascaded sliding mode observers for descriptor linear systems," Mathematical Problems in Engineering, vol. 2012, Article ID 623426, 20 pages, 2012.

[9] J. Wu, H. R. Karimi, and P. Shi, "Network-based $H_{\infty}$ output feedback control for uncertain stochastic systems," Information Sciences, vol. 232, pp. 397-410, 2013.

[10] H. R. Karimi, "Observer-based mixed $H_{2} / H_{\infty}$ control design of linear systems with time-varying delays: an LMI approach," International Journal of Control, Automation and Systems, vol. 6, no. 1, pp. 1-14, 2008.

[11] M. Chadi and H. R. Karimi, "Robust observer design for unknown inputs Takagi-Sugeno models," IEEE Transactions on Fuzzy Systems, vol. 21, no. 1, pp. 158-164, 2013.

[12] S. Zhou and W. Zhang, "Discrete-time indefinite stochastic LQ control via SDP and LMI methods," Journal of Applied Mathematics, vol. 2012, Article ID 638762, 14 pages, 2012.

[13] T. Zou, "Offset-free strategy by double-layered linear model predictive control," Journal of Applied Mathematics, vol. 2012, Article ID 808327, 14 pages, 2012.

[14] H. Zhang, J. Cao, and W. Jiang, "Controllability criteria for linear fractional differential systems with state delay and impulses," Journal of Applied Mathematics, vol. 2013, Article ID 146010, 9 pages, 2013. 
[15] C.-J. Wang and J.-S. Chiou, "A stability condition with delaydependence for a class of switched large-scale time-delay systems," Journal of Applied Mathematics, vol. 2013, Article ID 360170, 7 pages, 2013.

[16] M. S. Mahmoud, Robust Control and Filtering for Time-Delay Systems, vol. 5 of Control Engineering (New York), Marcel Dekker, New York, NY, USA, 2000.

[17] T. Chen and B. A. Francis, Optimal Sample-Data Control Systems, Springer, New York, NY, USA, 1995.

[18] D. Li and Y. Xi, "Constrained feedback robust model predictive control for polytopic uncertain systems with time delays," International Journal of Systems Science, vol. 42, no. 10, pp. 16511660, 2011.

[19] L. S. Shieh, J. S. H. Tsai, and S. R. Lian, "Determining continuous-time state equations from discrete-time state equations via the principal qth root method," IEEE Transactions on Automatic Control, vol. 31, no. 5, pp. 454-457, 1986.

[20] F. R. Ganmacher, The Theory of Matrices II, Chelsea, New York, NY, USA, 1974

[21] R. Nikoukhah, A. S. Willsky, and B. C. Levy, "Boundary-value descriptor systems: well-posedness, reachability and observability," International Journal of Control, vol. 46, no. 5, pp. 1715$1737,1987$.

[22] S. L. Campbell, Singular Systems of Differential Equations II, Pitman, New York, NY, USA, 1982.

[23] L. S. Shieh, Y. T. Tsay, and C. T. Wang, "Matrix sector functions and their applications to systems theory," IEE Proceedings D: Control Theory and Applications, vol. 131, no. 5, pp. 171-181, 1984.

[24] J. S. H. Tsai, L. S. Shieh, and R. E. Yates, "Fast and stable algorithms for computing the principal nth root of a complex matrix and the matrix sector function," Computers and Mathematics with Applications, vol. 15, no. 11, pp. 903-913, 1988.

[25] W.-M. Wang, S.-M. Guo, and L.-S. Shieh, "Discretization of cascaded continuous-time controllers for state and input delayed systems," International Journal of Systems Science, vol. 31, no. 3, pp. 287-296, 2000.

[26] S.-M. Guo, W. Wang, and L.-S. Shieh, "Discretisation of two degree-of-freedom controller and system with state, input and output delays," IEE Proceedings-Control Theory and Applications, vol. 147, no. 1, pp. 87-96, 2000.

[27] S.-M. Guo, L.-S. Shieh, G. Chen, and C.-F. Lin, "Effective chaotic orbit tracker: a prediction-based digital redesign approach," IEEE Transactions on Circuits and Systems I, vol. 47, no. 11, pp. 1557-1570, 2000.

[28] M. Hou, P. Zítek, and R. J. Patton, "An observer design for linear time-delay systems," IEEE Transactions on Automatic Control, vol. 47, no. 1, pp. 121-125, 2002.

[29] F. L. Lewis, Optimal Control, John Wiley \& Sons, New York, NY, USA, 1986.

[30] K. Ogata, Discrete-Time Control Systems, Prentice-Hall, Englewood Cliffs, NJ, USA, 1987.

[31] K. Watanabe and T. Ouchi, "An observer of systems with delays in state variables," International Journal of Control, vol. 41, no. 1, pp. 217-229, 1985.

[32] J. Leyva-Ramos and A. E. Pearson, "Asymptotic modal observer for linear autonomous time lag systems," IEEE Transactions on Automatic Control, vol. 40, no. 7, pp. 1291-1294, 1995.

[33] J. S.-H. Tsai, N.-T. Hu, P.-C. Yang, S.-M. Guo, and L.-S. Shieh, "Modeling of decentralized linear observer and tracker for a class of unknown interconnected large-scale sampled-data nonlinear systems with closed-loop decoupling property," Computers and Mathematics with Applications, vol. 60, no. 3, pp. 541$562,2010$.

[34] N. T. Hu, J. S. H. Tsai, S. M. Guo, L. S. Shieh, and Y. Chen, "Loworder multi-rate linear time-invariant decentralized trackers using the new observer-based sub-optimal method for unknown sampled-data nonlinear time-delay system with closed-loop decoupling," Optimal Control Applications and Methods, vol. 32, no. 4, pp. 433-475, 2011. 


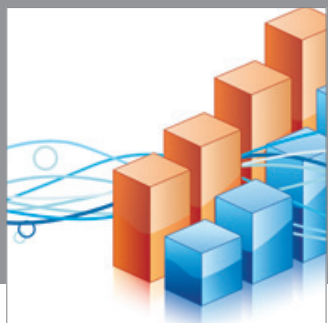

Advances in

Operations Research

mansans

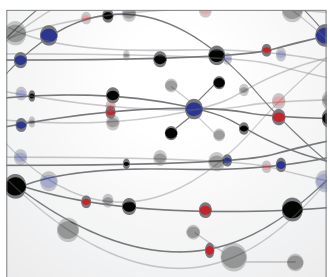

The Scientific World Journal
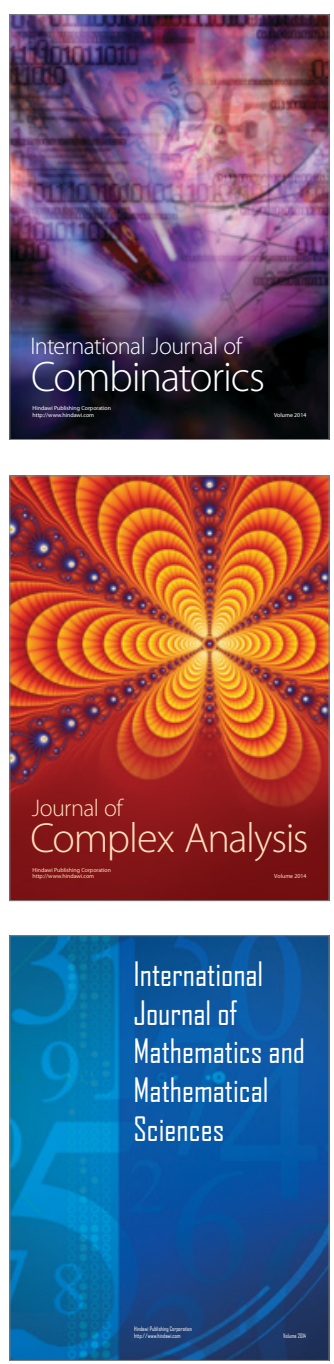
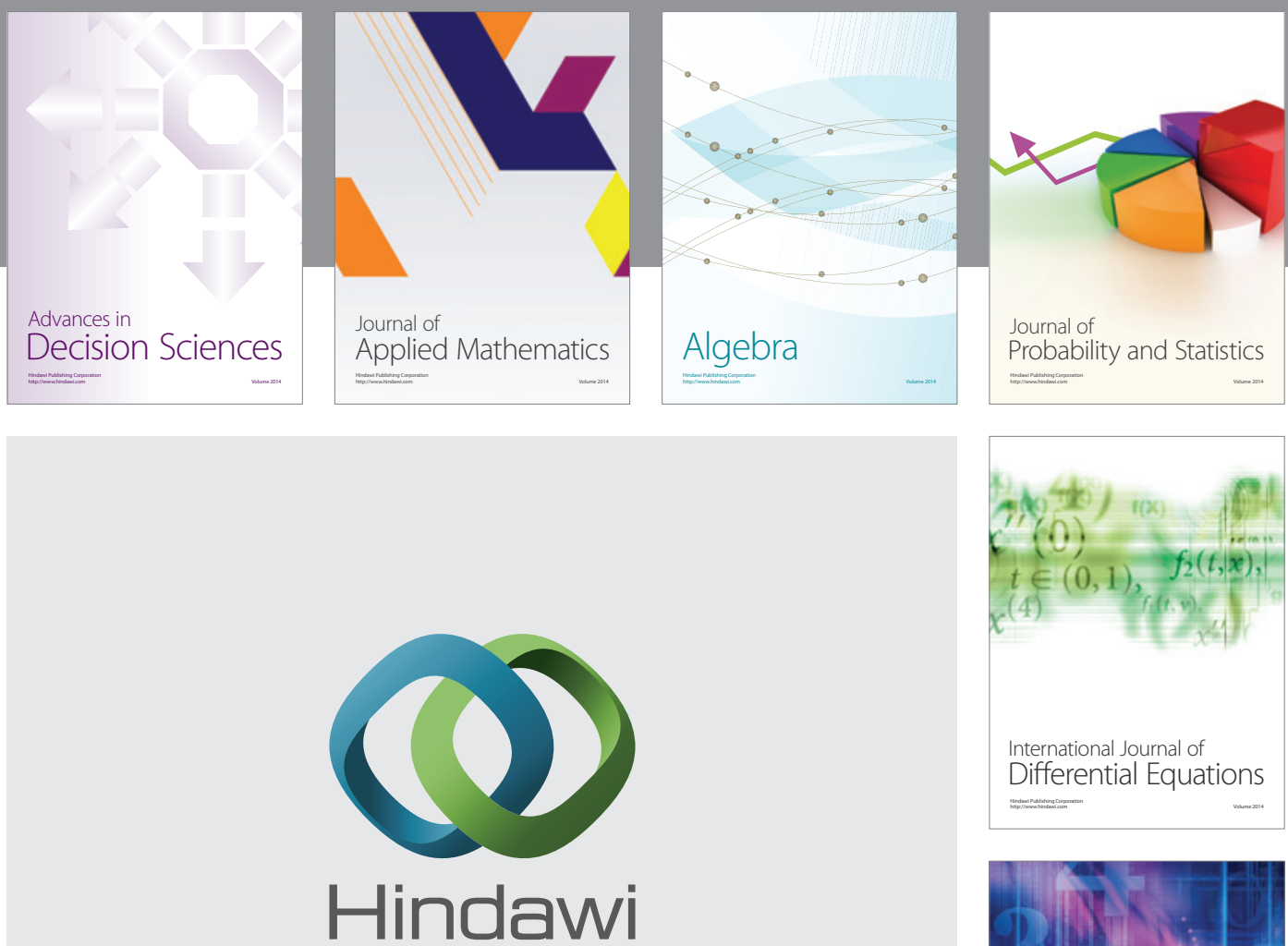

Submit your manuscripts at http://www.hindawi.com
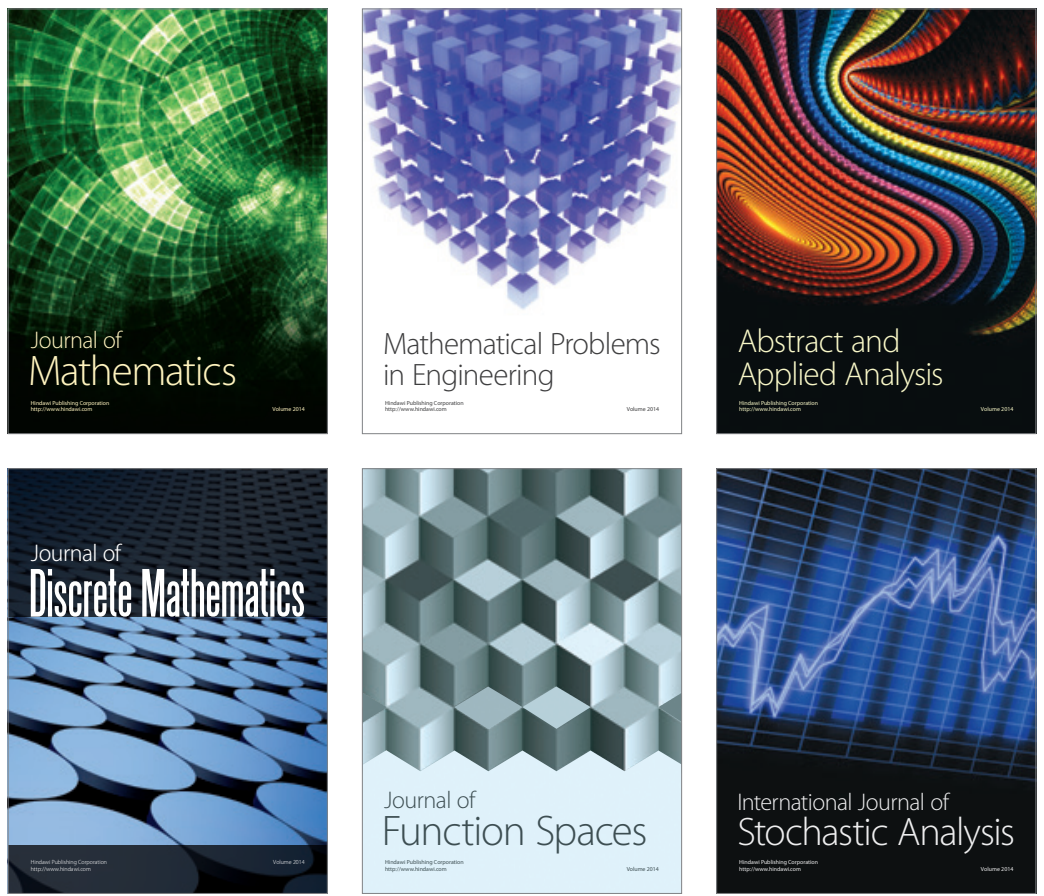

Journal of

Function Spaces

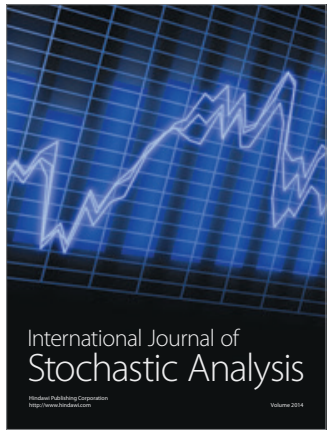

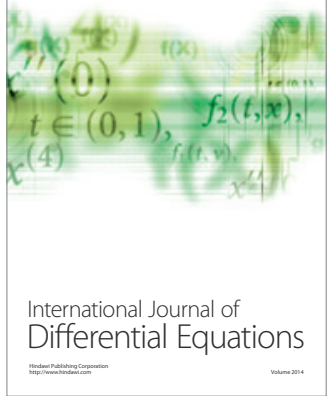
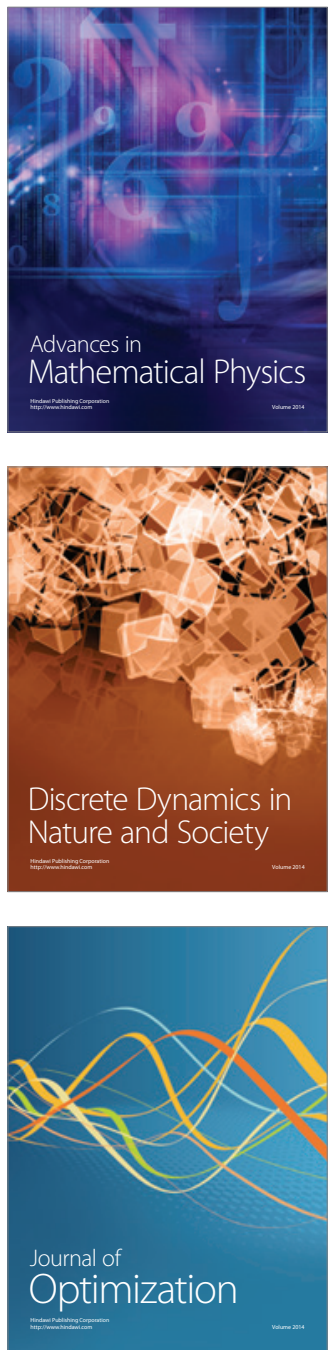